\title{
Supporting Information for \\ Potential Energy Function for Fentanyl-Based Opioid Pain Killers
}

\author{
Samo Lešnik ${ }^{1,2}$, Milan Hodošček ${ }^{3}$, Urban Bren², Christoph Stein ${ }^{4}$, \\ and Ana-Nicoleta Bondar ${ }^{1, *}$
}

${ }^{1}$ Freie Universität Berlin, Department of Physics, Theoretical Molecular Biophysics Group, Arnimallee 14, D-14195 Berlin, Germany

2University of Maribor, Faculty of Chemistry and Chemical Engineering,

Smetanova ulica 17, SI-2000 Maribor, Slovenia

${ }^{3}$ National Institute of Chemistry Slovenia, Theory Department, Hajdrihova 19,

SI-1000 Ljubljana, Slovenia

${ }^{4}$ Charité-Universitätsmedizin Berlin, Campus Benjamin Franklin, Department of

Experimental Anaesthesiology, Hindenburgdamm 30, 12203 Berlin, Germany

Supporting information contains:

- Supplementary Figures S1-S8

- Supplementary Tables S1-S2

- Topology and parameter file information for Sets $A, B$ and $C$ for fentanyl

- Topology and parameter file information for NFEPP

*Corresponding author e-mail address: nbondar@zedat.fu-berlin.de 
a

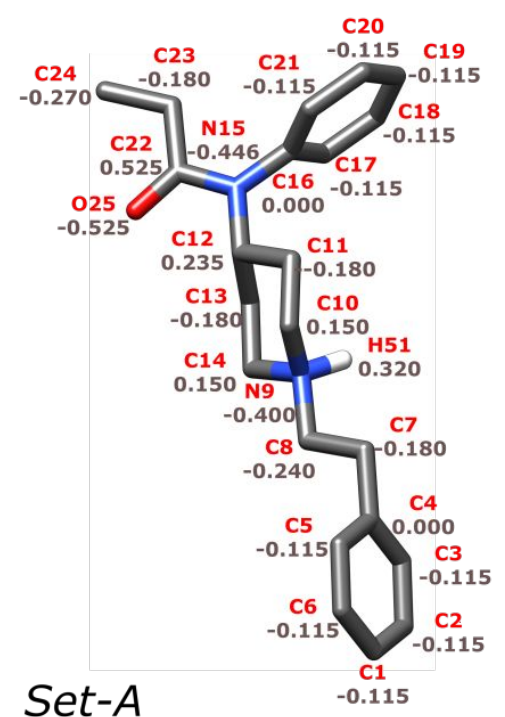

b

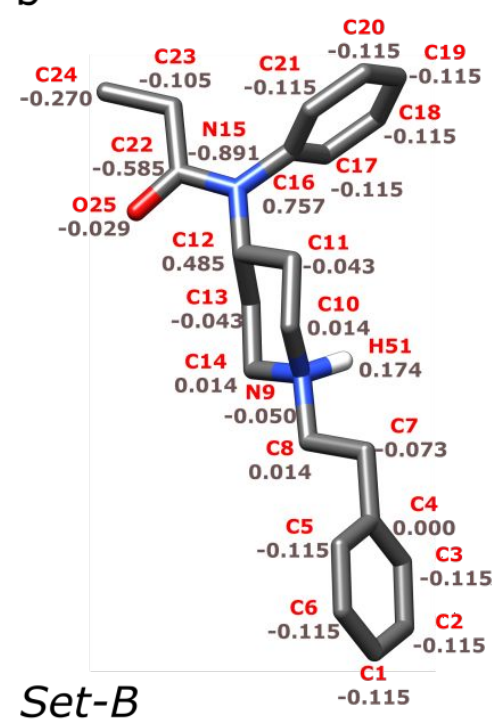

C

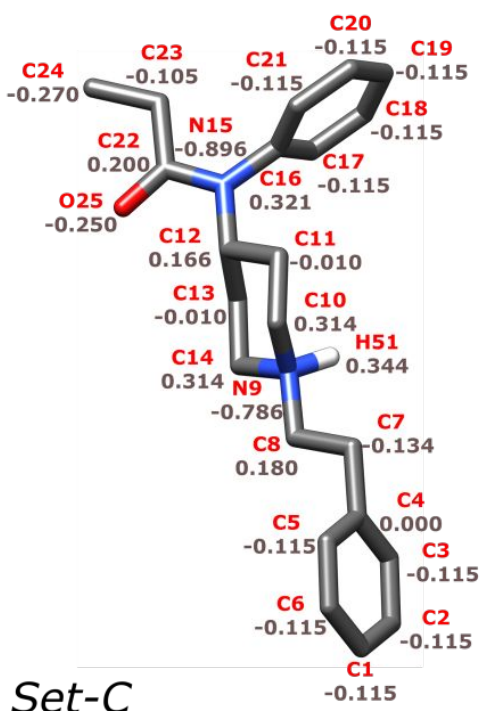

Figure S1. Three sets of partial charge parameters we prepared for fentanyl. According to the CGenFF protocol, all non-polar hydrogen atoms are assigned a charge of $0.090 e$, and all aromatic hydrogen atoms are assigned a charge of $0.115 e$. These hydrogen atoms are not shown for clarity.
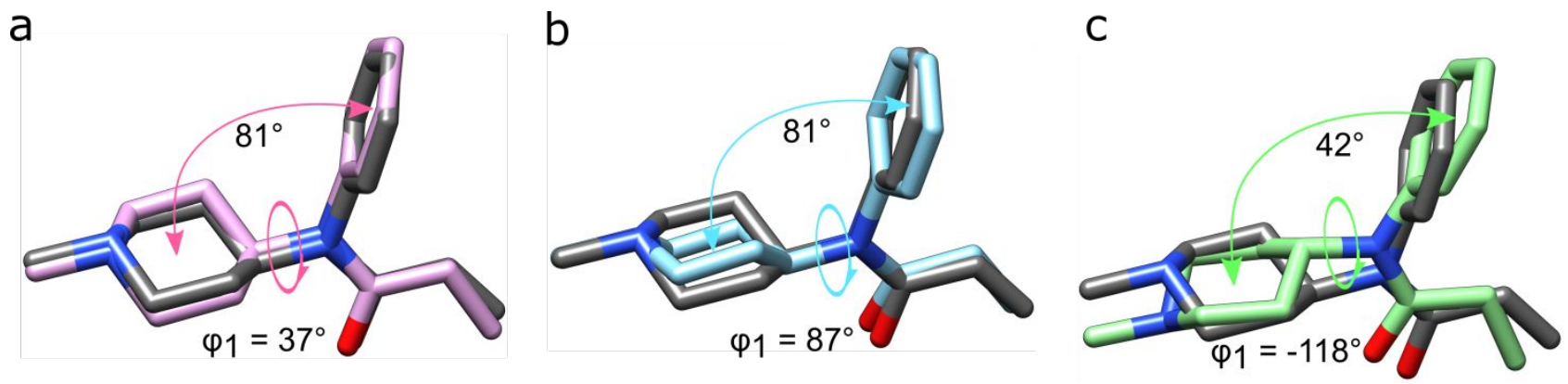

Figure S2. Overlap between MP2 structures and starting crystal structure of fentanyl with Fr-1 removed. The crystal structure is shown with carbon atoms colored gray, and structures from MP2 PES profiles are shown with carbon atoms colored pink, cyan, or green. (a) Structure of fentanyl at the local minimum at $\varphi_{1}=37^{\circ}$ (pink) overlapped with the crystal structure from ref. 1. (b) Fentanyl at the local minimum at $\varphi_{1}=87^{\circ}$ in the PES (cyan) overlapped with the crystal structure from ref. 1. (c) Fentanyl at the minimum at $\varphi 1$ $=-118^{\circ}$ (green) in the PES, overlapped with the crystal structure from ref 1 . In the crystal structure from ref 1 ., the angle between the two ring planes is $81.8^{\circ}$. At the minima shown in panels $\mathrm{a}, \mathrm{b}$, and $\mathrm{c}$, this angle is $81.1^{\circ}, 80.8^{\circ}$, and $42.1^{\circ}$, respectively. 


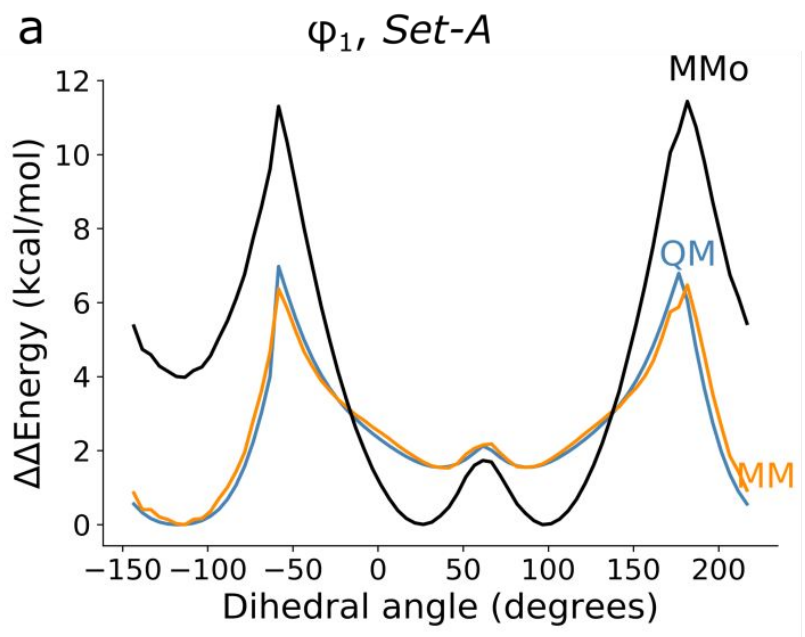

b $\quad \varphi_{2}$, Set- $A$
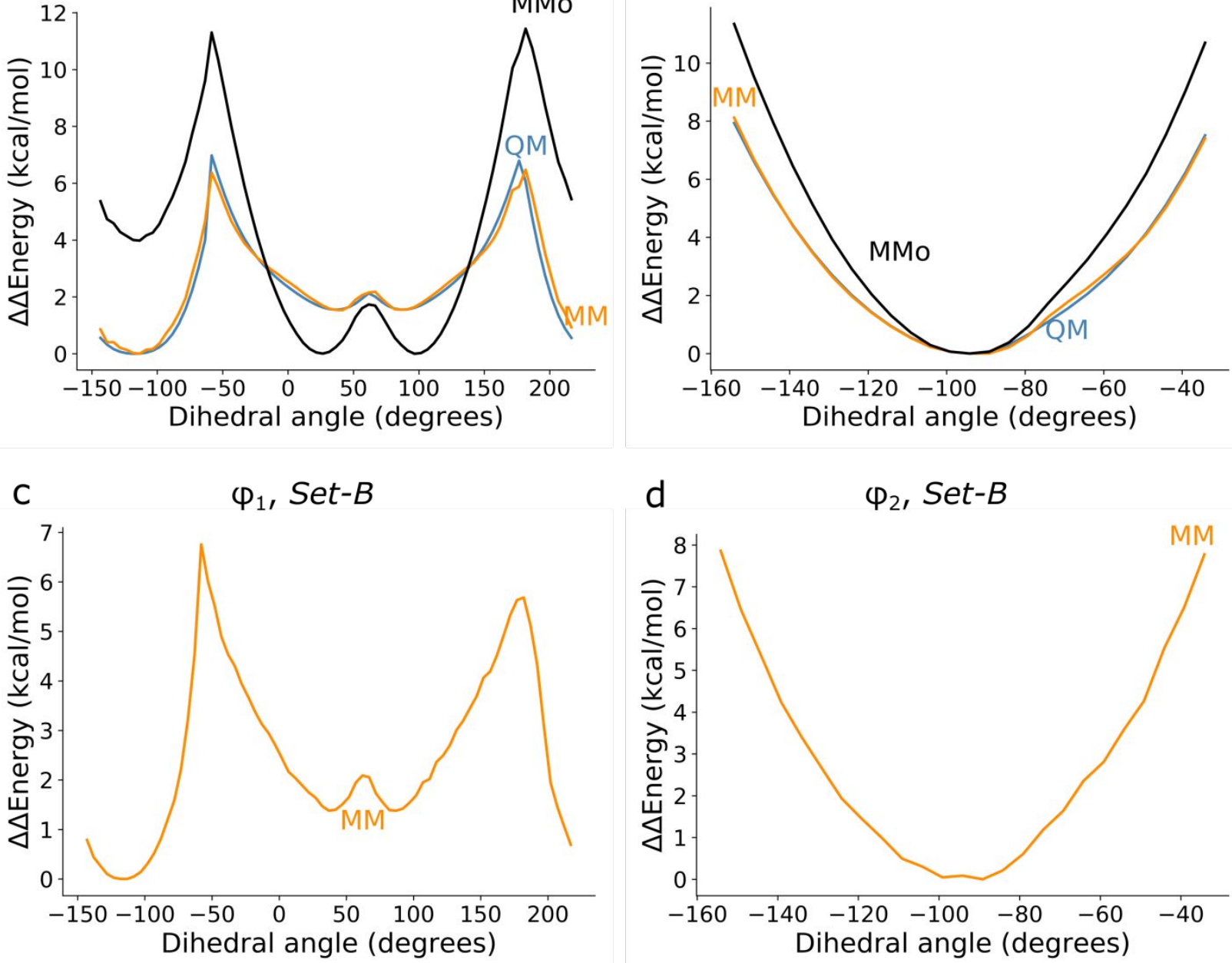

Figure S3. PES for dihedral angles, computed with MP2 and with MM. MP2 profiles are colored cyan. Profiles computed with the original MM and with the optimized MM parameters are colored black and orange, respectively. (a-b) PES scans for dihedral angles $\varphi_{1}$ and $\varphi_{2}$; for the optimized MM profile we used parameters Set-A. (c-d) PES scans for dihedral angles $\varphi_{1}$ and $\varphi_{2}$; for the optimized MM profile we used parameters SetB.

a

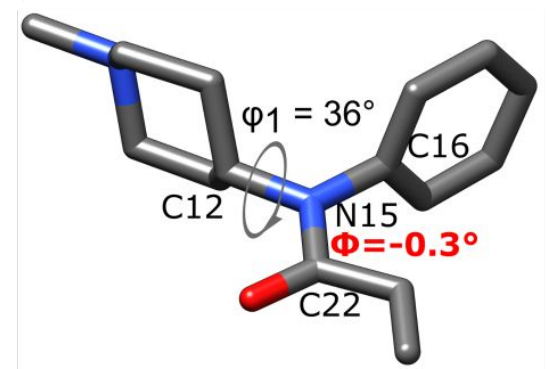

b

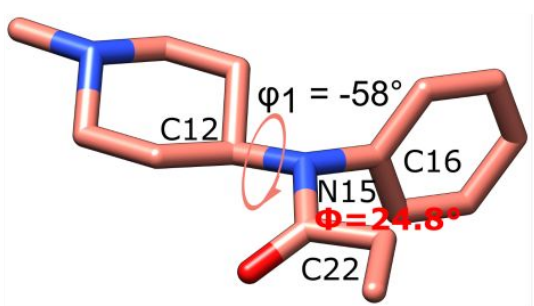

C

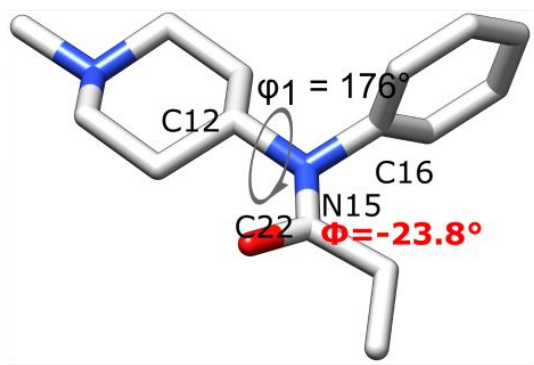

Figure S4. Molecular graphics illustrating the structure of fentanyl at energy maximum along the PES of dihedral angle $\varphi_{1}$. (a) Crystal structure of fentanyl is from ref. 1. In the crystal structure (a) $\mathrm{N} 15$ is in plane with the three carbon atoms to which it is covalently bound, and the dihedral angle $\Phi$, which describes the out of plane movement of $\mathrm{N} 15$, is $-0.3^{\circ}$. (b) Geometry of fentanyl at $\varphi_{1}=$ $58^{\circ}$ in the MP2 PES. (c) Geometry of fentanyl at $\varphi_{1}=176^{\circ}$ in the MP2 PES. At the two energy maxima depicted in panels b and c, N15 is no longer in plane with the three carbon atoms to which it is covalently bound, and $\Phi$ has values of $\sim 24-25^{\circ}$. 


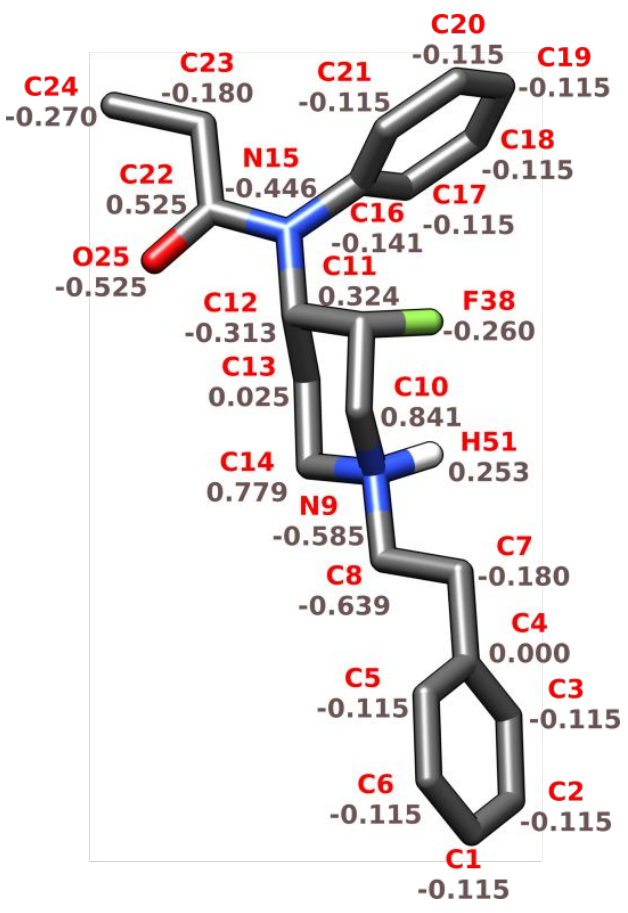

Figure S5. Partial atomic charges for 3-fluorofentanyl. According to CGenFF, all non-polar hydrogens are assigned a charge of $0.090 e$, and all aromatic hydrogens are assigned a charge of $0.115 e$. These hydrogen atoms are not shown for clarity. 

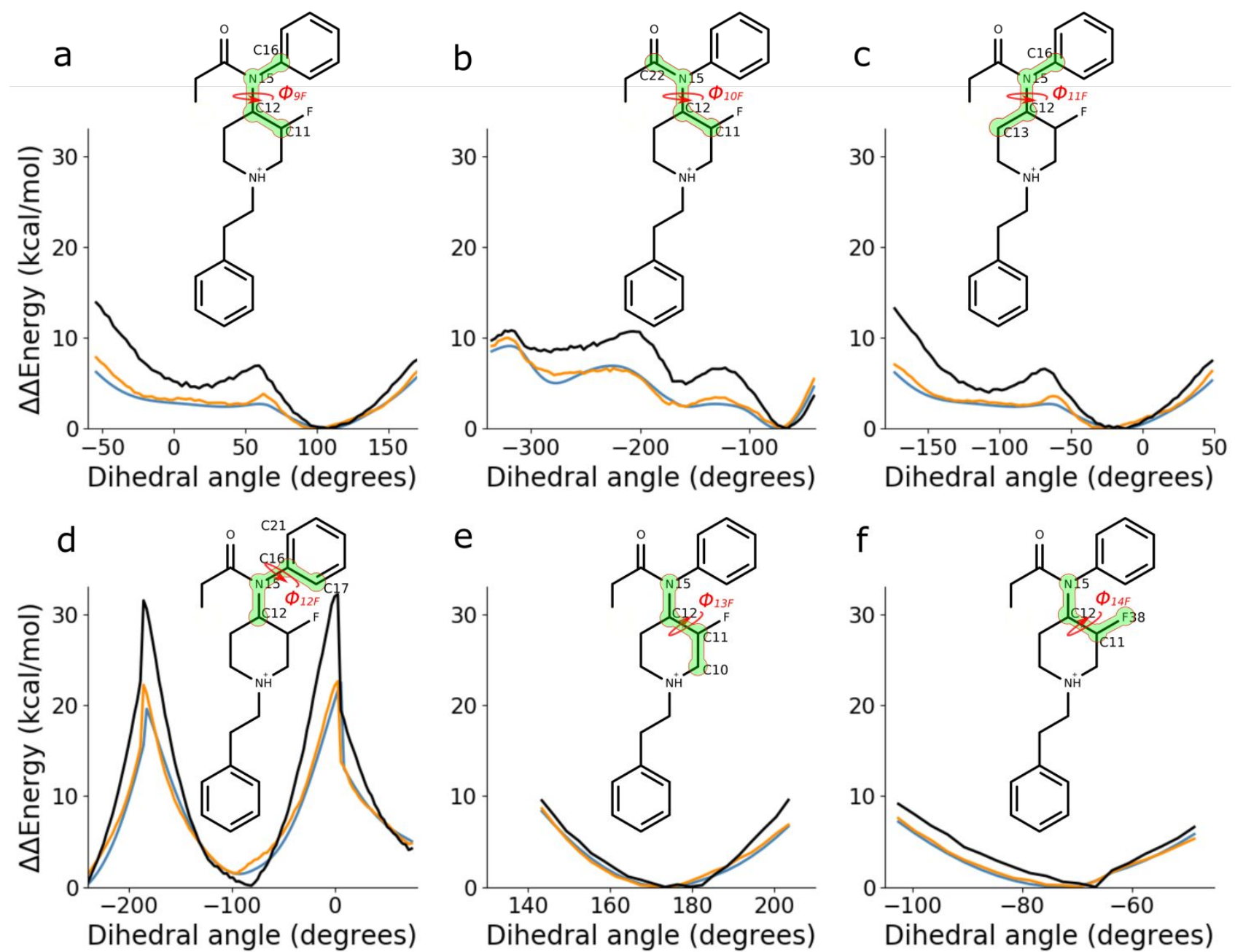

Figure S6. PES profiles for dihedral angles in the linkage between the 3-fluoropiperidine fragment (Fr-2F) and amidobenze (Fr-3). ParamChem indicated high penalty scores for all these six dihedral angles. (a-f) PES profiles computed with MP2 (cyan), optimized MM parameters (blue), and original MM parameters (orange). 

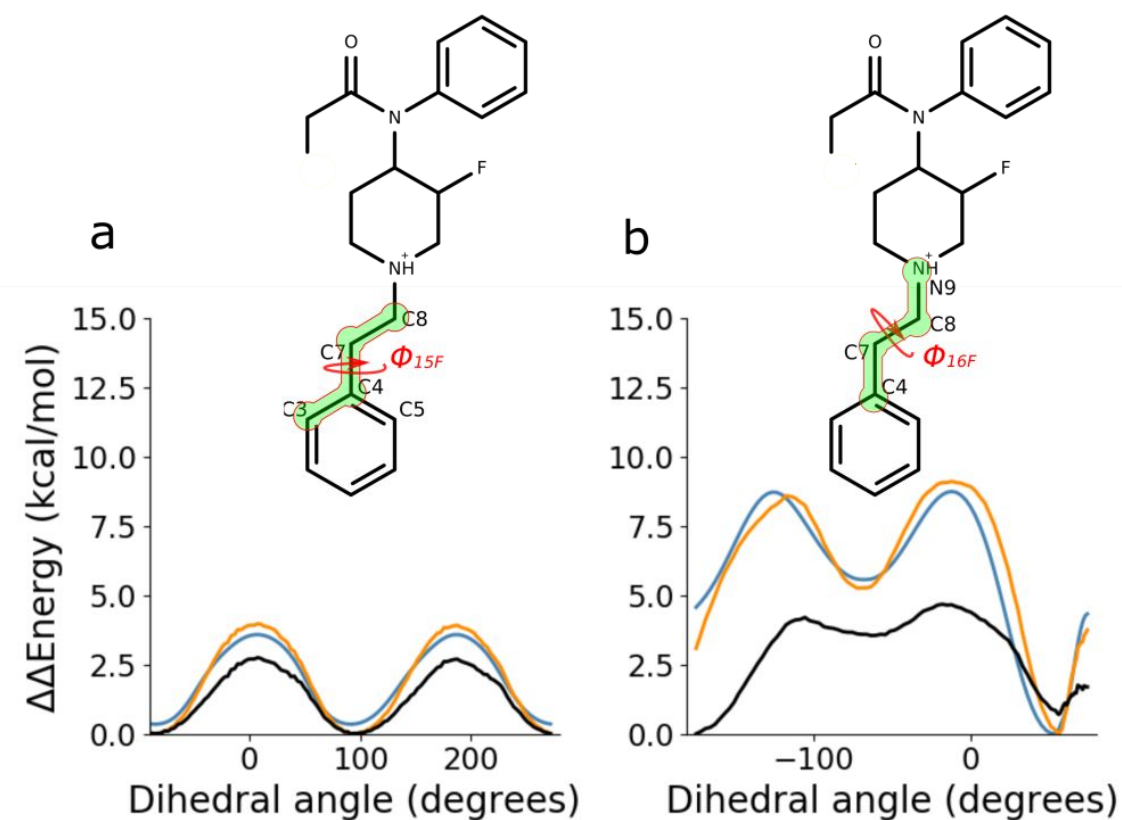

$C$
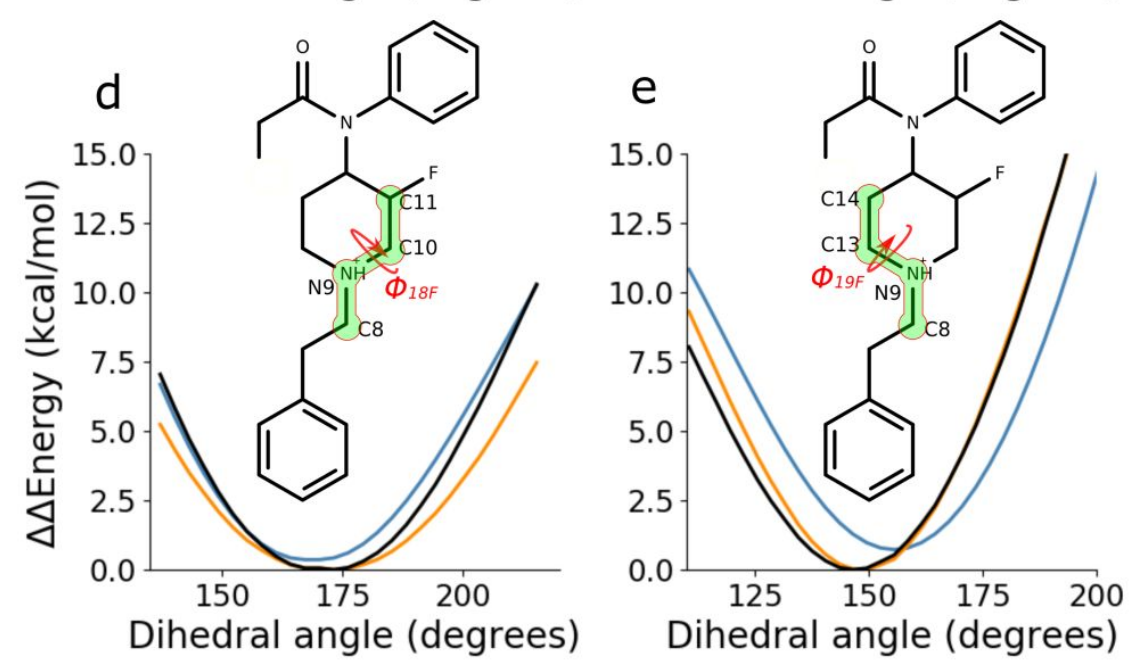

Figure S7. PES profiles for dihedral angles at the linkage between the 3-fluoropiperidine fragment (Fr-2F) and ethylbenezne (Fr-1) of NFEPP. ParamChem indicated high-penalty scores for these dihedral angles. (a-e) PES profiles computed with MP2, optimized MM, and original MM parameters are shown in cyan, blue, and orange, respectively.

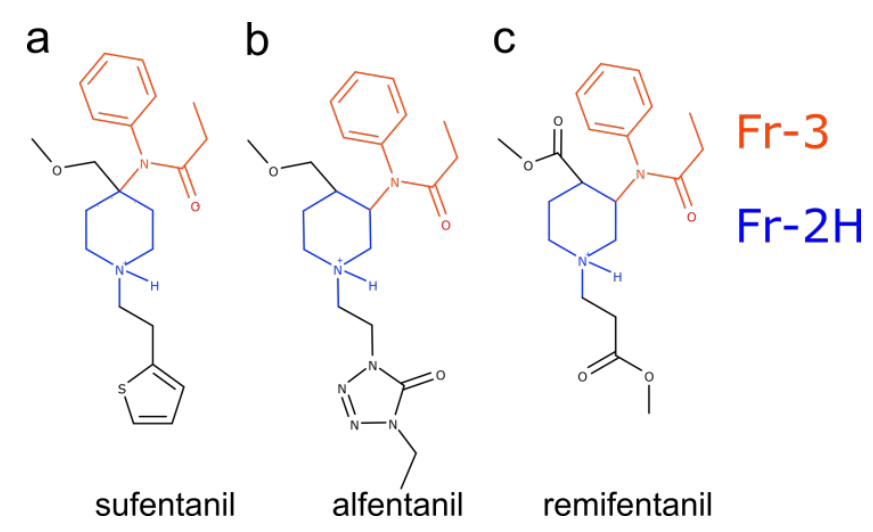

Figure S8. Schematic representation of fentanyl derivatives. (a-c) Schematic representation of sufentanil, alfentanil, and remifentanil. 
Table S1. HF and MM water interaction energies and distances obtained for the purpose of partial charge calculations of Set-B and Set-C fentanyl parameters.

\begin{tabular}{cc|cc|cc|cc}
\hline $\begin{array}{c}\text { Interaction } \\
\text { site }\end{array}$ & $\begin{array}{c}\text { Water interaction } \\
\text { type }\end{array}$ & $\begin{array}{c}\Delta E_{H F} \\
{[k c a l / m o l]}\end{array}$ & $\begin{array}{c}R_{H F} \\
{[\AA]}\end{array}$ & $\begin{array}{c}\Delta E_{M M} \\
{[\mathrm{kcal} / \mathrm{mol}]} \\
(\text { Set B) }\end{array}$ & $\begin{array}{c}R_{M M} \\
{[\AA]} \\
(\text { Set } B)\end{array}$ & $\begin{array}{c}\Delta E_{M M}[\mathrm{kcal} / \mathrm{mol}] \\
(\text { Set B) }\end{array}$ & $\begin{array}{c}R_{M M}[\AA] \\
(\text { Set } C)\end{array}$ \\
\hline O25 & acceptor & -5.78 & 2.97 & -5.81 & 2.81 & -5.87 & 2.77 \\
H31 & donor & -7.00 & 2.47 & -6.98 & 2.54 & -6.66 & 2.57 \\
H33 & donor & -7.50 & 2.33 & 7.52 & 2.49 & -7.30 & 2.51 \\
H35 & donor & -8.46 & 2.26 & -8.48 & 2.46 & -8.44 & 2.46 \\
H37 & donor & -6.31 & 2.47 & -6.35 & 2.54 & -5.55 & 2.59 \\
H39 & donor & -4.51 & 2.39 & -4.59 & 2.54 & -4.66 & 2.57 \\
H51 & donor & -14.08 & 1.93 & -14.03 & 1.86 & -14.14 & 1.84 \\
\hline
\end{tabular}

Table S2. HF and MM water interaction energies and distances obtained for the purpose of partial charge calculations of NFEPP Fr-2F fragment parameters.

\begin{tabular}{cc|cc|cc}
\hline Interaction site & Water interaction type & $\Delta E_{H F}[\mathrm{kcal} / \mathrm{mol}]$ & $\Delta R_{H F}[\AA]$ & $\Delta E_{M M}[\mathrm{kcal} / \mathrm{mol}]$ & $\Delta R_{M M}[\AA]$ \\
\hline H36 & donor & -9.53 & 2.19 & -9.53 & 2.39 \\
H37 & donor & -7.53 & 2.24 & -7.53 & 2.41 \\
F38 & acceptor & -0.80 & 2.95 & -0.79 & 2.84 \\
H40 & donor & -6.48 & 2.33 & -6.48 & 2.48 \\
H43 & donor & -9.15 & 2.24 & -9.16 & 2.40 \\
H51 & donor & -12.86 & 1.96 & -12.88 & 1.82 \\
\hline
\end{tabular}


* Topology file (.rtf) for FENTANYL (FNT); parameterization by total analogy of partial charges; Set A

* Prepared by Dr. Samo Lesnik, 2019, A. N. Bondar Group

* Potential energy function for fentanyl-based pain killers - Samo Lešnik, Milan Hodošček, Urban Bren, Christoph Stein, and AnaNicoleta Bondar

\begin{tabular}{|c|c|c|c|c|}
\hline RESI FNT & & 1.000 & & \\
\hline GROUP & & & & \\
\hline ATOM C1 & CG2R61 & -0.115 & $!$ & \\
\hline АТОМ H26 & HGR 61 & 0.115 & ! & \\
\hline GROUP & & & & \\
\hline ATOM C2 & CG2R61 & -0.115 & $!$ & \\
\hline АТОМ H27 & HGR 61 & 0.115 & $!$ & \\
\hline GROUP & & & & \\
\hline ATOM C3 & CG2R61 & -0.115 & $!$ & \\
\hline АTOM H28 & HGR 61 & 0.115 & $!$ & \\
\hline GROUP & & & & \\
\hline ATOM C4 & CG2R61 & 0.000 & $!$ & \\
\hline GROUP & & & & \\
\hline ATOM C5 & CG2R61 & -0.115 & $!$ & \\
\hline ATOM H29 & HGR 61 & 0.115 & $!$ & \\
\hline GROUP & & & & \\
\hline ATOM C 6 & CG2R61 & -0.115 & $!$ & \\
\hline АТОМ Н30 & HGR 61 & 0.115 & $!$ & \\
\hline GROUP & & & & \\
\hline $\mathrm{ATOM} \quad \mathrm{C} 7$ & CG321 & -0.180 & $!$ & CONNECTION \\
\hline АТОМ H31 & HGA2 & 0.090 & $!$ & \\
\hline АТОМ H32 & HGA 2 & 0.090 & $!$ & \\
\hline GROUP & & & & \\
\hline ATOM C8 & CG324 & 0.240 & $!$ & CONNECTION \\
\hline АTOM H33 & HGA 2 & 0.090 & $!$ & \\
\hline АТОM H34 & HGA 2 & 0.090 & $!$ & \\
\hline ATOM N9 & NG3P1 & -0.400 & $!$ & \\
\hline АTOM H51 & HGP 2 & 0.320 & $!$ & \\
\hline ATOM C10 & CG324 & 0.150 & $!$ & \\
\hline АТОМ H35 & HGA2 & 0.090 & $!$ & \\
\hline ATOM H36 & HGA2 & 0.090 & $!$ & \\
\hline ATOM C14 & CG324 & 0.150 & $!$ & \\
\hline ATOM H42 & HGA2 & 0.090 & $!$ & \\
\hline ATOM H43 & HGA2 & 0.090 & $!$ & \\
\hline GROUP & & & & \\
\hline АTOM C11 & CG321 & -0.180 & $!$ & \\
\hline АТОМ H37 & HGA 2 & 0.090 & $!$ & \\
\hline ATOM H38 & HGA 2 & 0.090 & $!$ & \\
\hline GROUP & & & & \\
\hline ATOM C13 & CG321 & -0.180 & $!$ & \\
\hline ATOM H4O & HGA 2 & 0.090 & $!$ & \\
\hline ATOM H41 & HGA 2 & 0.090 & $!$ & \\
\hline
\end{tabular}




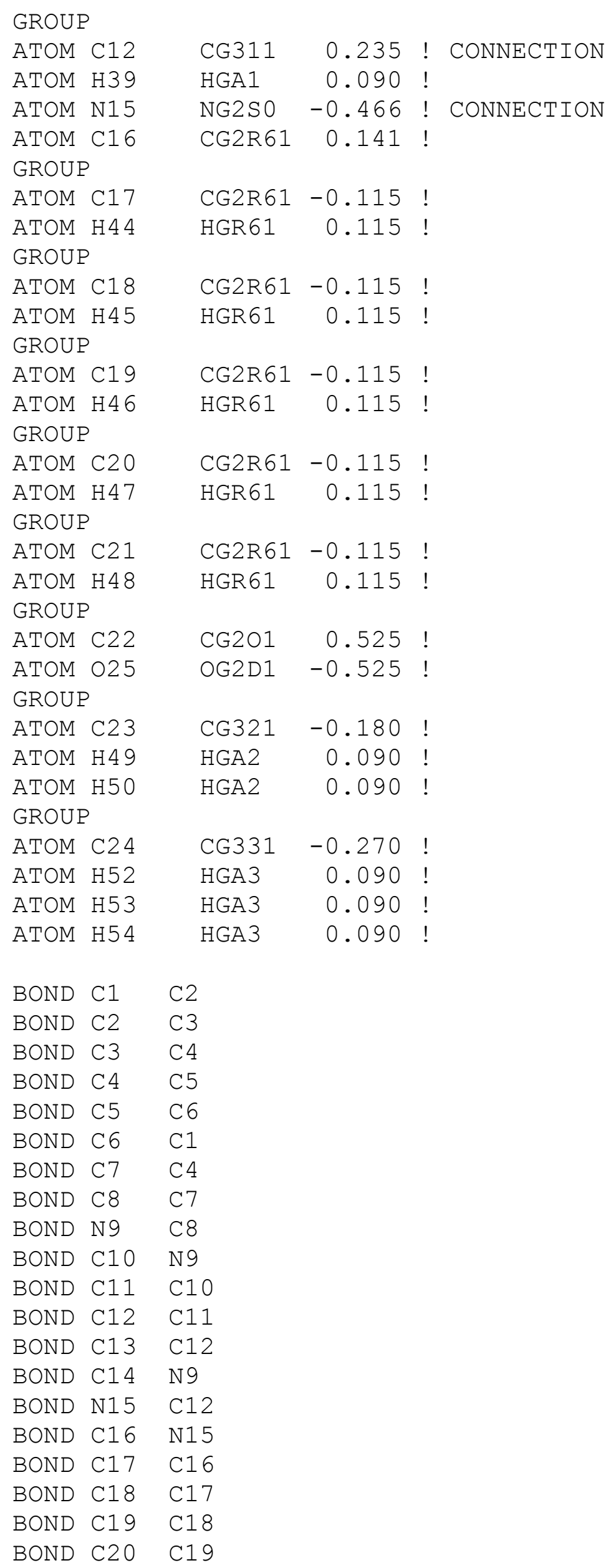




\begin{tabular}{|c|c|c|c|}
\hline BOND & C21 & C16 & \\
\hline BOND & C22 & N15 & \\
\hline BOND & C23 & C22 & \\
\hline BOND & C24 & C23 & \\
\hline BOND & 025 & C22 & \\
\hline BOND & H2 6 & $\mathrm{C} 1$ & \\
\hline BOND & H2 7 & $\mathrm{C} 2$ & \\
\hline BOND & H2 8 & C3 & \\
\hline BOND & H2 9 & C5 & \\
\hline BOND & $\mathrm{H} 3 \mathrm{O}$ & C6 & \\
\hline BOND & H31 & C7 & \\
\hline BOND & H32 & C7 & \\
\hline BOND & H33 & C8 & \\
\hline BOND & H3 4 & C8 & \\
\hline BOND & H35 & $\mathrm{C} 10$ & \\
\hline BOND & H3 6 & $\mathrm{C} 10$ & \\
\hline BOND & H37 & $\mathrm{C} 11$ & \\
\hline BOND & H38 & $\mathrm{C} 11$ & \\
\hline BOND & H39 & C12 & \\
\hline BOND & $\mathrm{H} 4 \mathrm{O}$ & $\mathrm{C} 13$ & \\
\hline BOND & H 41 & $\mathrm{C} 13$ & \\
\hline BOND & $\mathrm{H} 42$ & $\mathrm{C} 14$ & \\
\hline BOND & $\mathrm{H} 43$ & C14 & \\
\hline BOND & $\mathrm{H} 44$ & C17 & \\
\hline BOND & H4 5 & C18 & \\
\hline BOND & H4 6 & C19 & \\
\hline BOND & H 47 & $\mathrm{C} 20$ & \\
\hline BOND & H 48 & C21 & \\
\hline BOND & H 49 & C23 & \\
\hline BOND & H5O & C23 & \\
\hline BOND & H51 & N9 & \\
\hline BOND & C13 & C14 & \\
\hline BOND & $\mathrm{C} 20$ & C21 & \\
\hline BOND & H52 & C24 & \\
\hline BOND & H5 3 & C24 & \\
\hline BOND & H5 4 & C24 & \\
\hline IMPR & C22 & C23 & N15 \\
\hline
\end{tabular}

\footnotetext{
* Parameter file (.prm) for FENTANYL (ENT); parameterization by total analogy of partial charges; set A

* Prepared by Dr. Samo Lesnik, 2019, A. N. Bondar Group

* Potential energy function for fentanyl-based pain killers - Samo Lešnik, Milan Hodošček, Urban Bren, Christoph Stein and AnaNicoleta Bondar

*
}

BONDS

CG2O1 CG321 250.00 1.4900! PROT Ala Dipeptide (5/91)

CG2O1 NG2S0 430.00 1.3500 ! DMA, Dimethylacetamide, xxwy

CG2O1 OG2D1 620.00 1.2300 ! PROT Peptide geometry,

condensed phase (LK) 


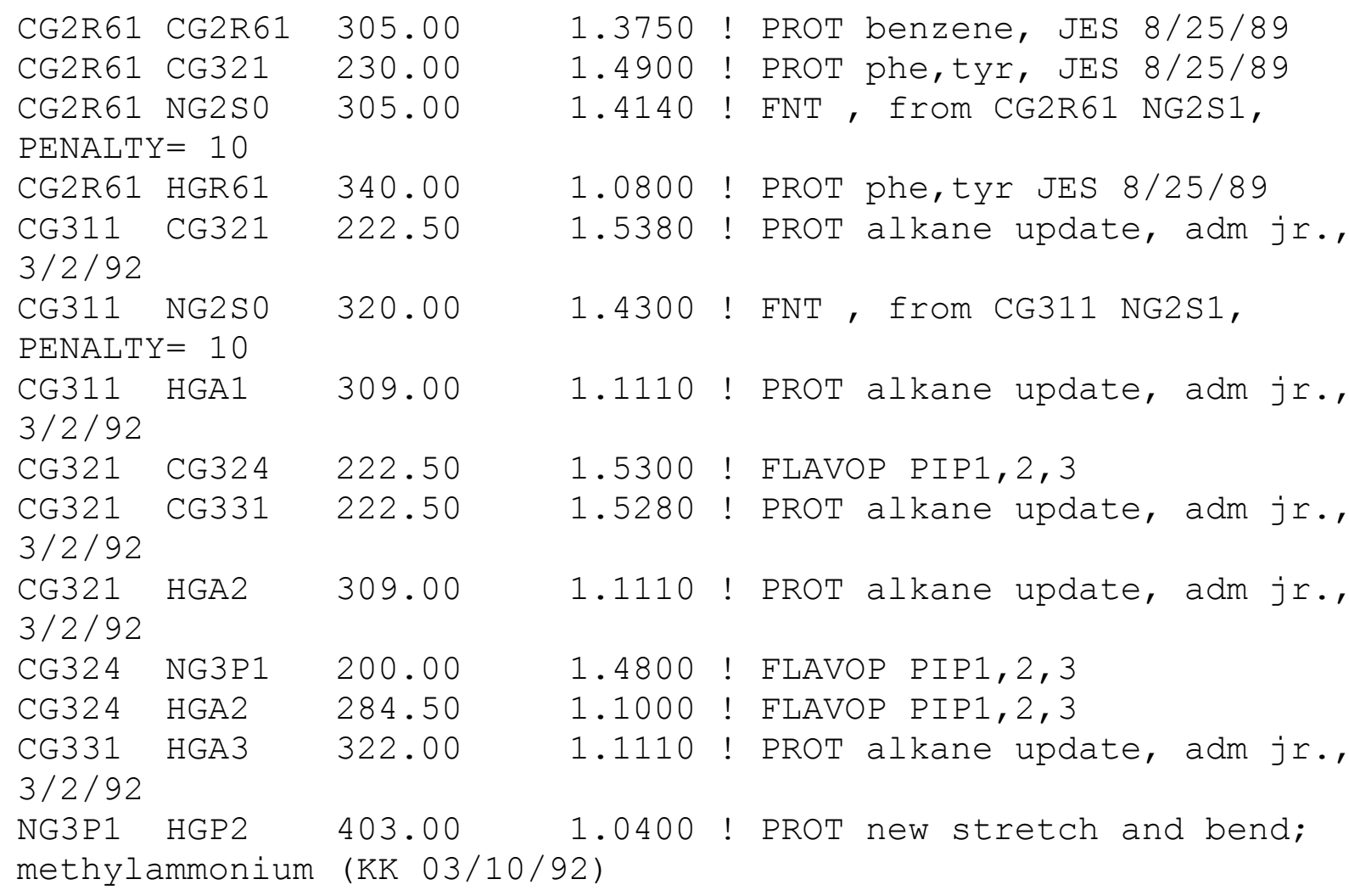

\footnotetext{
ANGLES

CG321 CG2O1 NG2SO $20.00 \quad 112.50$ ! PROT ACProNH2, ProNH2, ACPrONHCH3 RLD 4/23/93

CG321 CG2O1 OG2D1 80.00 121.00 ! PROT Alanine Dipeptide ab initio calc's (LK) NG2SO CG2O1 OG2D1 80.00 124.00 ! DMF, Dimethylformamide, $\begin{array}{lllllll}\text { XXWY } & & & & & & \\ \text { CG2R61 CG2R61 CG2R61 } & 40.00 & 120.00 & 35.00 & 2.41620 & \text { ! PROT }\end{array}$ JES $8 / 25 / 89$

CG2R61 CG2R61 CG321 45.80 120.00 ! EBEN, ethylbenzene, modified by kevo for improved transferability CG2R61 CG2R61 NG2S0 $40.00 \quad 120.00 \quad 35.00 \quad 2.41620$ ! FNT , from CG2R61 CG2R61 NG2S1, PENALTY $=1$ $\begin{array}{lllllll}\text { CG2R61 CG2R61 HGR61 } 30.00 & 120.00 & 22.00 & 2.15250 & \text { ! PROT }\end{array}$ JES 8/25/89 benzene $\begin{array}{llllllll}\text { CG321 CG311 } & \text { CG321 } & 58.35 & 113.50 & 11.16 & 2.56100 & \text { ! LIPID }\end{array}$ glycerol

CG321 CG311 NG2S0 70.00 108.00 ! FNT, from CG321 CG311 NG2S1, PENALTY= 1 CHANGED BY SAMO: eq. dist from 113.50

$\begin{array}{llllllll}\text { CG321 CG311 HGA1 } & 34.50 & 110.10 & 22.53 & 2.17900 & \text { ! PROT }\end{array}$ alkane update, adm jr., 3/2/92

NG2SO CG311 HGA1 $48.00 \quad 102.00$ ! FNT, from NG2S1 CG311 HGA1, PENALTY= 1 !!! CHANGED BY SAMO: eq. dist 108.00

CG2O1 CG321 CG331 52.00 108.00 ! PROT adm jr. 4/05/91, for asn, asp, gln, glu and cters
} 


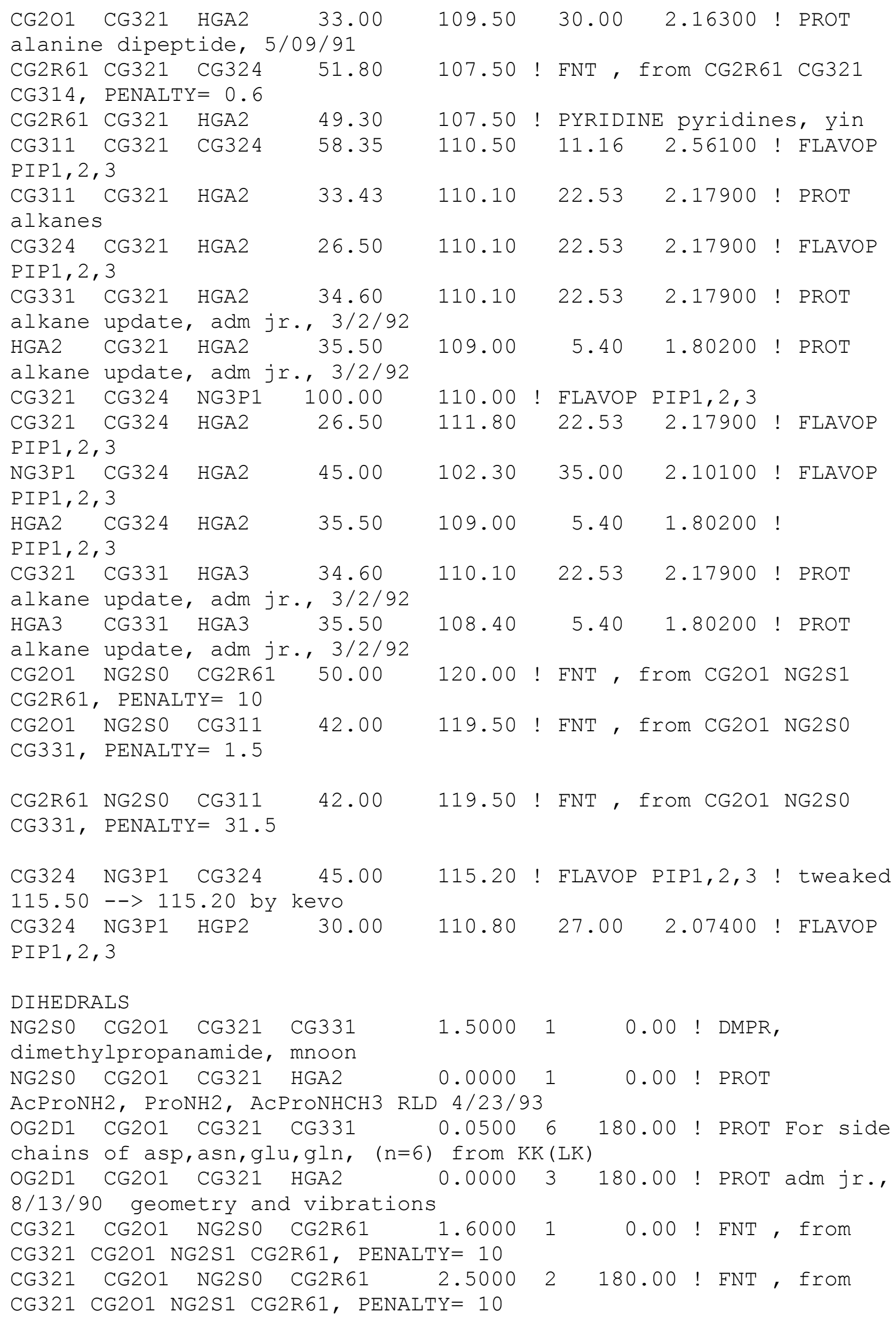


CG321 CG2O1 NG2S0 CG311 2.6000 2 180.00 ! FNT ， from CG321 CG2O1 NG2SO CG331, PENALTY $=1.5$

OG2D1 CG2O1 NG2S0 CG2R61 2.5000 2 180.00 ! FNT, from OG2D1 CG2O1 NG2S1 CG2R61, PENALTY $=10$

OG2D1 CG2O1 NG2S0 CG311 2.6000 2 180.00 ! FNT， from OG2D1 CG2O1 NG2SO CG331, PENALTY $=1.5$

CG2R61 CG2R61 CG2R61 CG2R61 3.1000 $2 \quad 180.00$ ! PROT JES $8 / 25 / 89$

CG2R61 CG2R61 CG2R61 CG321 3.1000 2 180.00 ! PROT JES

8/25/89 toluene and ethylbenzene

CG2R61 CG2R61 CG2R61 NG2S0 3.100

CG2R61 CG2R61 CG2R61 NG2S1, PENALTY= 1

CG2R61 CG2R61 CG2R61 HGR61 4.20002180 .00 ! PROT JES

$8 / 25 / 89$ benzene

CG321 CG2R61 CG2R61 HGR61 2.4000 $2 \quad 180.00$ ! PROT JES

8/25/89 toluene and ethylbenzene Kenno: 4.2 -> 2.4

NG2S0 CG2R61 CG2R61 HGR61 2.4000 2 180.00 ! FNT ，from

NG2S1 CG2R61 CG2R61 HGR61, PENALTY = 1

$\begin{array}{llllllll}\text { HGR61 CG2R61 CG2R61 HGR61 2.4000 } 2 & 180.00 & \text { ! PROT JES }\end{array}$

$8 / 25 / 89$ benzene

CG2R61 CG2R61 CG321 CG324 0.65002180 .00 ! FNT ， from CG2R61 CG2R61 CG321 CG314, PENALTY=0.6 ! before 0.23002

180.00

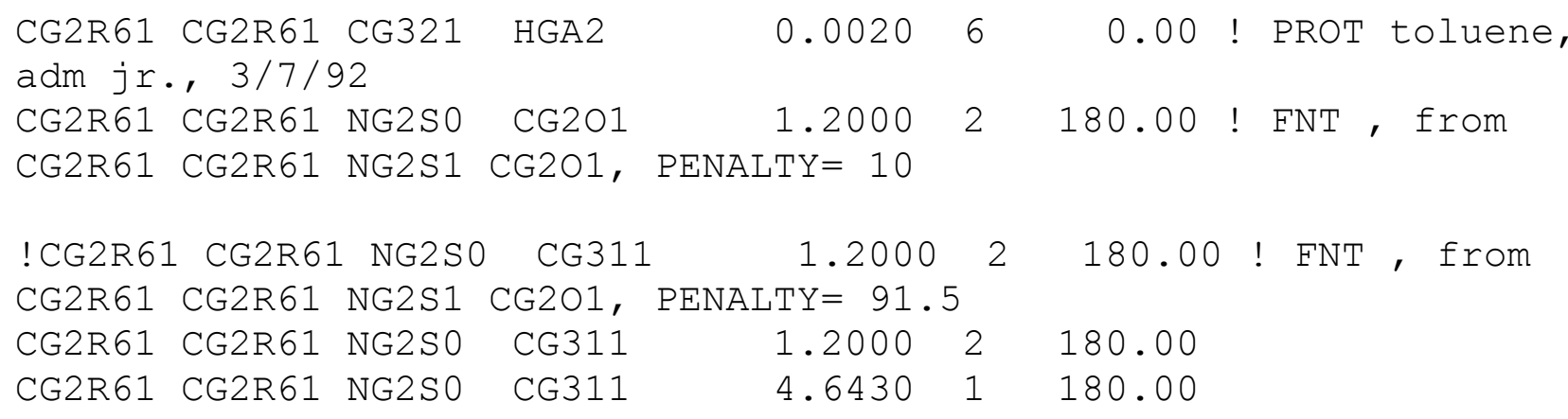

$\begin{array}{llllllll}\text { CG321 CG311 CG321 } & \text { CG324 } & 0.2000 & 3 & 0.00\end{array}$ 4-Methyl piperidine Glu Acid CDCA Amide, cacha reset to default by kevo

CG321

NG2SO CG311 CG321 $\mathrm{CG} 324$

$0.1950 \quad 1$

$0.2000 \quad 3$

NG2S1 CG311 CG321 CG324, PENALTY $=1$

NG2SO CG311 CG321 HGA2 $0.2000 \quad 3$

NG2S1 CG311 CG321 HGA2, PENALTY $=1$

HGA1 CG311 CG321 CG324 0.195030 .00 ! G4MP, 010H03,

cacha

HGA1 CG311 CG321 HGA2 $0.1950 \quad 3$

CG321 CG311 NG2S0 CG2O1 $1.8000 \quad 1$

CG321 CG311 NG2S1 CG2O1, PENALTY= 10

!CG321 CG311 NG2S0 CG2R61

$1.8000 \quad 1$

CG321 CG311 NG2S1 CG2O1, PENALTY $=40$

$\begin{array}{lllllll}\text { CG321 CG311 NG2S0 } & \text { CG2R61 } & 3.8360 & 1 & 0.00\end{array}$

$\begin{array}{lllllll}\text { CG321 CG311 NG2S0 } & \text { CG2R61 } & 3.8360 & 1 & 0.00\end{array}$

$0.00 ! \mathrm{NA}$, sugar

0.00 ! FNT, from

$0.00 !$ FNT, from

$0.00 ! \mathrm{NA}$, sugar

0.00 ! FNT, from 


$\begin{array}{lllllll}\text { CG321 } & \text { CG311 } & \text { NG2S0 } & \text { CG2R61 } & 0.6680 & 3 & 0.00 \\ \text { CG321 } & \text { CG311 } & \text { NG2S0 } & \text { CG2R61 } & 0.0490 & 6 & 180.00\end{array}$

HGA1 CG311 NG2SO CG2O1 $0.00001 \quad 0.00$ ! FNT， from

HGA1 CG311 NG2S1 CG2O1, PENALTY= 10

HGA1 CG311 NG2S0 CG2R61 $0.00001 \quad 0.00$ ! FNT , from

HGA1 CG311 NG2S1 CG2O1, PENALTY $=40$

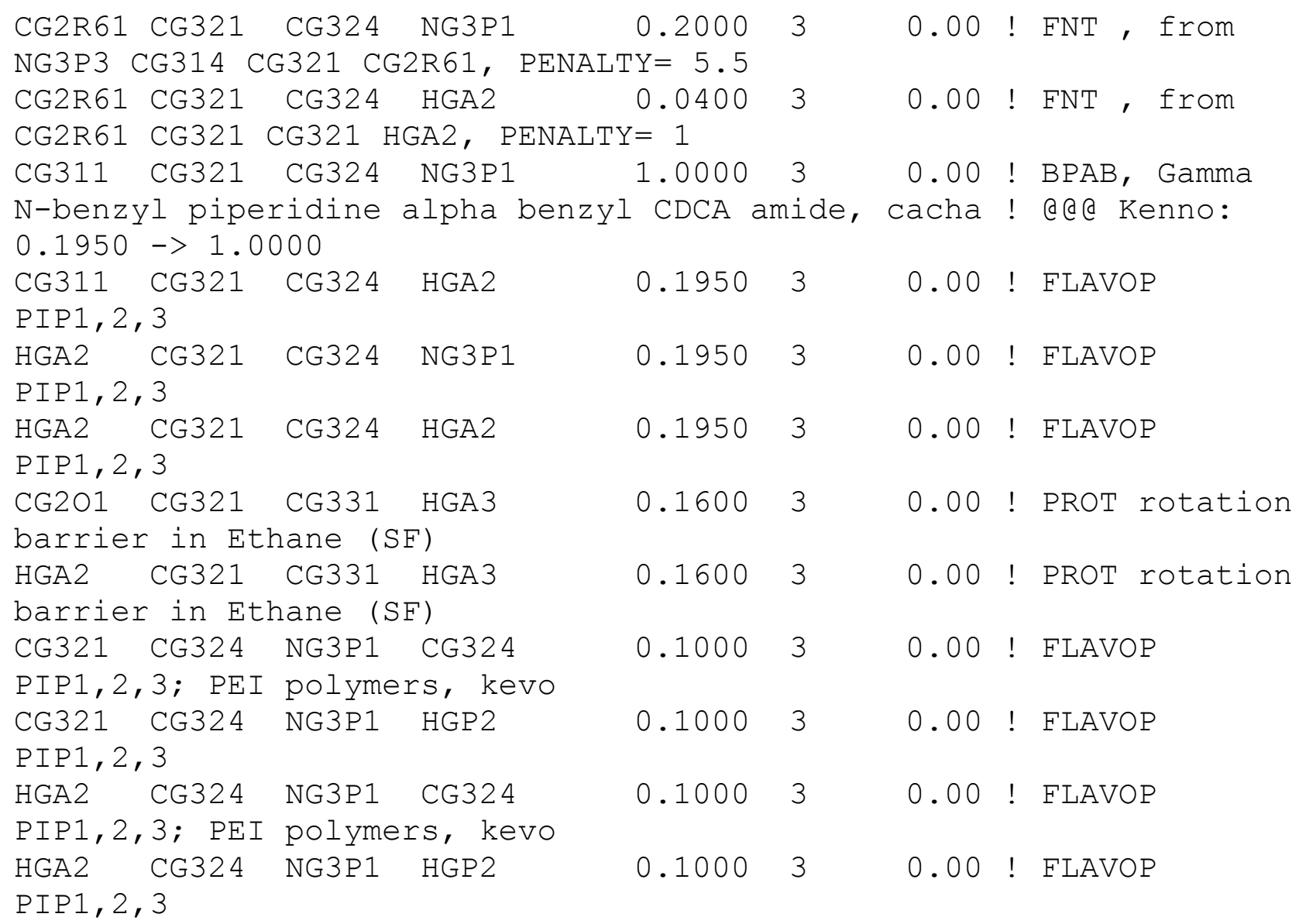

\section{IMPROPERS}

$\begin{array}{llllllll}\text { CG2O1 CG321 NG2S0 OG2D1 } & 120.0000 & 0 & 0.00 & \text { ! PROT NMA }\end{array}$ Vibrational Modes (LK) WILDCARD 


\section{Set-B parameters for fentanyl}

* Topology file (.rtf) for FENTANYL (FNT); parameterization by best water interaction partial charges; Set B

* Prepared by Dr. Samo Lesnik, 2019, A. N. Bondar Group

* Potential energy function for fentanyl-based pain killers - Samo Lešnik, Milan Hodošček, Urban Bren, Christoph Stein and AnaNicoleta Bondar

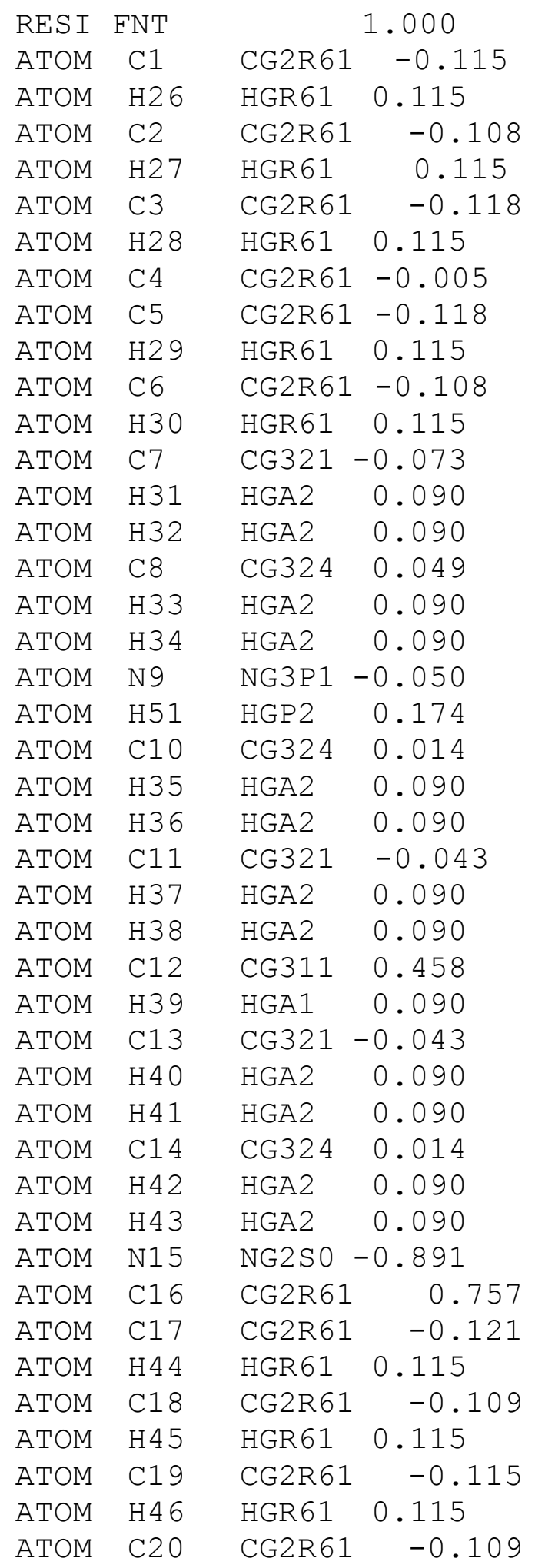




\begin{tabular}{|c|c|c|}
\hline ATOM & H4 7 & HGR61 0.115 \\
\hline ATOM & $\mathrm{C} 21$ & CG2R61 \\
\hline АTOM & H4 8 & HGR61 0.115 \\
\hline ATOM & $\mathrm{C} 22$ & $\mathrm{CG} 201-0.585$ \\
\hline ATOM & $\mathrm{C} 23$ & CG321-0.105 \\
\hline ATOM & H4 9 & $\begin{array}{ll}\text { HGA } 2 & 0.090\end{array}$ \\
\hline АTOM & H5O & 0.090 \\
\hline АTOM & $\mathrm{C} 24$ & CG331 -0.270 \\
\hline ATOM & H52 & 0.090 \\
\hline ATOM & H53 & 0.090 \\
\hline ATOM & H5 4 & 0.090 \\
\hline ATOM & 025 & OG2D1 -0.029 \\
\hline BOND & $\mathrm{C} 1$ & $\mathrm{C} 2$ \\
\hline $\mathrm{BOND}$ & $\mathrm{C} 2$ & $\mathrm{C} 3$ \\
\hline BOND & $\mathrm{C} 3$ & $\mathrm{C} 4$ \\
\hline BOND & $\mathrm{C} 4$ & $\mathrm{C} 5$ \\
\hline BOND & $\mathrm{C} 5$ & $\mathrm{C} 6$ \\
\hline BOND & $\mathrm{C} 6$ & $\mathrm{C} 1$ \\
\hline BOND & C7 & $\mathrm{C} 4$ \\
\hline BOND & $\mathrm{C} 8$ & $\mathrm{C} 7$ \\
\hline BOND & N9 & $\mathrm{C} 8$ \\
\hline $\mathrm{BOND}$ & $\mathrm{C} 10$ & N9 \\
\hline BOND & $\mathrm{C} 11$ & $\mathrm{C} 10$ \\
\hline BOND & $\mathrm{C} 12$ & $\mathrm{C} 11$ \\
\hline BOND & $\mathrm{C} 13$ & $\mathrm{C} 12$ \\
\hline BOND & $\mathrm{C} 14$ & N9 \\
\hline BOND & N15 & $\mathrm{C} 12$ \\
\hline BOND & C16 & N15 \\
\hline BOND & C17 & C16 \\
\hline BOND & C18 & C17 \\
\hline BOND & C19 & C18 \\
\hline BOND & $\mathrm{C} 20$ & C19 \\
\hline BOND & C21 & C16 \\
\hline BOND & C22 & N15 \\
\hline BOND & C23 & C22 \\
\hline BOND & C24 & C23 \\
\hline BOND & 025 & C22 \\
\hline BOND & H2 6 & $\mathrm{C} 1$ \\
\hline BOND & H27 & $\mathrm{C} 2$ \\
\hline BOND & H2 8 & C3 \\
\hline BOND & H2 9 & C5 \\
\hline BOND & $\mathrm{H} 3 \mathrm{O}$ & $\mathrm{C} 6$ \\
\hline BOND & H31 & C7 \\
\hline BOND & H32 & $\mathrm{C} 7$ \\
\hline BOND & H33 & C8 \\
\hline BOND & H34 & C8 \\
\hline BOND & H35 & $\mathrm{C} 10$ \\
\hline BOND & H36 & $\mathrm{C} 10$ \\
\hline BOND & H37 & $\mathrm{C} 11$ \\
\hline BOND & H38 & $\mathrm{C} 11$ \\
\hline BOND & H39 & $\mathrm{C} 12$ \\
\hline BOND & $\mathrm{H} 4 \mathrm{O}$ & $\mathrm{C} 13$ \\
\hline
\end{tabular}


BOND H41 C13

BOND H42 C14

BOND H43 C14

BOND H44 C17

BOND H45 C18

BOND H46 C19

BOND H47 C20

BOND H48 C21

BOND H49 C23

BOND H5O C23

BOND H51 N9

BOND $\mathrm{C} 13 \quad \mathrm{C} 14$

BOND C20 C21

BOND H52 C24

BOND H53 C24

BOND H54 C24

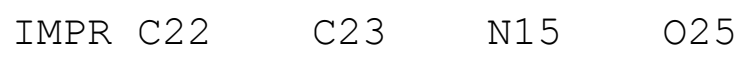


* Parameter (.prm) file for FENTANYL (FNT); parameterization by best water interaction partial charges; set B

* Prepared by Dr. Samo Lesnik, 2019, A. N. Bondar Group

* Potential energy function for fentanyl-based pain killers - Samo Lešnik, Milan Hodošček, Urban Bren, Christoph Stein and AnaNicoleta Bondar

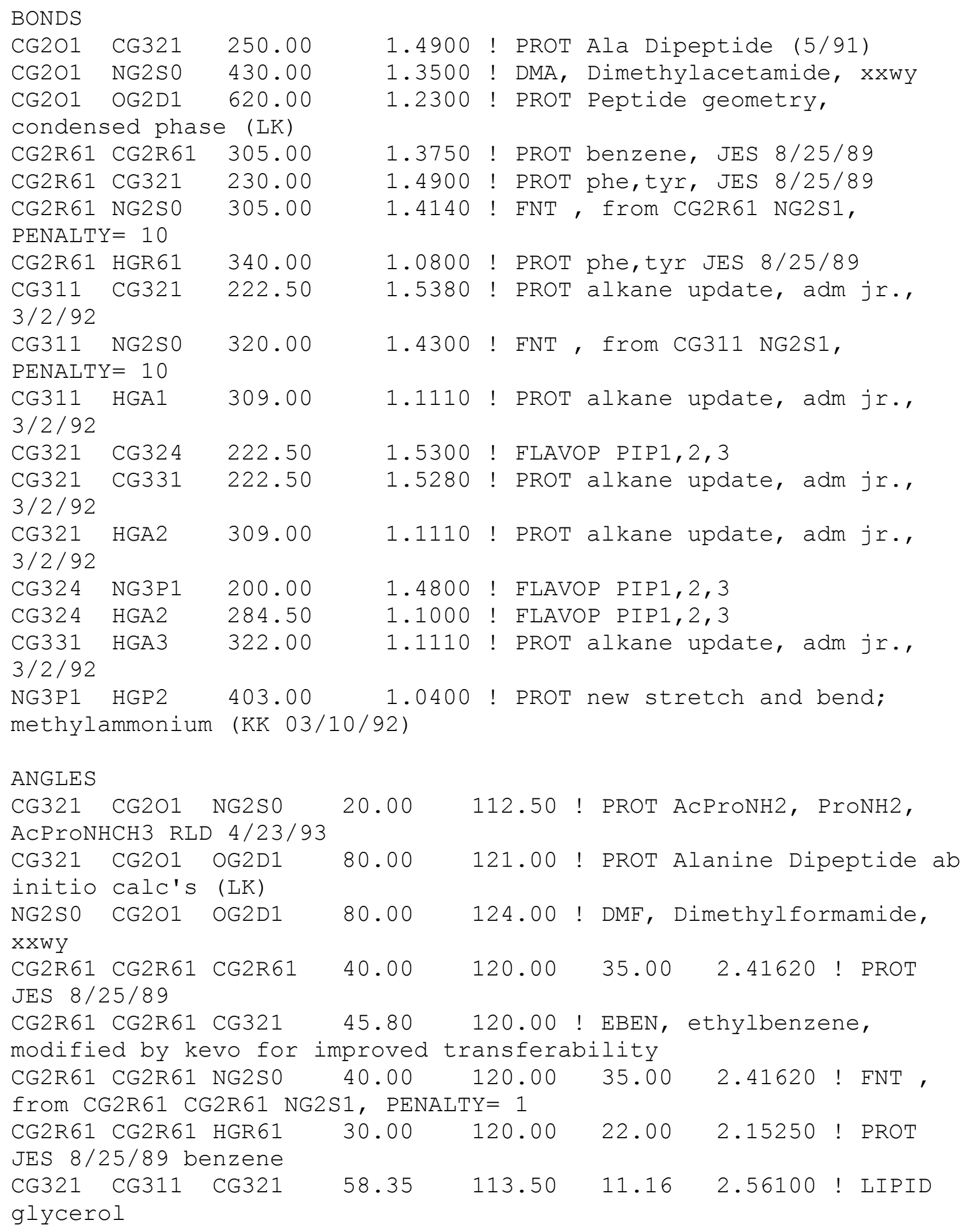


CG321 CG311 NG2S0 70.00 107.00 ! fnt_ga, from CG321 CG311 NG2S1, PENALTY= 1 ! CHANGED BY SAMO: eq. dist from 113.50

$\begin{array}{llllllll}\text { CG321 CG311 HGA1 } & 34.50 & 110.10 & 22.53 & 2.17900 & \text { ! PROT }\end{array}$ alkane update, adm jr., 3/2/92

NG2SO CG311 HGA1 48.00 102.00! fnt ga, from NG2S1 CG311 HGA1, PENALTY= 1 !!! CHANGED BY SAMO: eq. dist 108.00

CG2O1 CG321 CG331 52.00 108.00 ! PROT adm jr. 4/05/91, for asn, asp,gln, glu and cters

$\begin{array}{llllllll}\text { CG2O1 CG321 HGA2 } & 33.00 & 109.50 & 30.00 & 2.16300 & \text { ! PROT }\end{array}$

alanine dipeptide, 5/09/91

CG2R61 CG321 CG324 51.80

CG314, PENALTY $=0.6$

CG2R61 CG321 HGA2 49.30 107.50 ! PYRIDINE pyridines, yin

$\begin{array}{lllllll}\text { CG311 CG321 } & \text { CG324 } & 58.35 & 110.50 & 11.16 & 2.56100 & \text { ! FLAVOP }\end{array}$

PIP 1, 2, 3

$\begin{array}{llllllll}\text { CG311 CG321 HGA2 } & 33.43 & 110.10 & 22.53 & 2.17900 & \text { ! PROT }\end{array}$

alkanes

$\begin{array}{llllllll}\text { CG324 CG321 HGA2 } & 26.50 & 110.10 & 22.53 & 2.17900 & \text { ! FLAVOP }\end{array}$

PIP1, 2, 3

alkane update, adm jr., 3/2/92

HGA2 CG321 HGA2 35.50

alkane update, adm jr., 3/2/92

$\begin{array}{llll}\text { CG321 CG324 NG3P1 } & 100.00\end{array}$

$109.00 \quad 5.40 \quad 1.80200 \quad !$ PROT

CG321 CG324 HGA2 26.50

110.00 ! FLAVOP PIP1,2,3

PIP1, 2, 3

NG3P1 CG324 HGA2 45.00 $102.30 \quad 35.00 \quad 2.10100 \quad$ ! FLAVOP

$\mathrm{PIP} 1,2,3$

$\begin{array}{llllll}\text { HGA2 } & \text { CG324 HGA2 } & 35.50 \quad 109.00 & 5.40 & 1.80200 \quad \text { ! }\end{array}$

PIP1, 2, 3

$\begin{array}{llllllll}\text { CG321 CG331 HGA3 } & 34.60 & 110.10 & 22.53 & 2.17900 & \text { ! PROT }\end{array}$

alkane update, adm jr., 3/2/92

$\begin{array}{llllllll}\text { HGA3 } & \text { CG331 HGA3 } & 35.50 & 108.40 & 5.40 & 1.80200 & \text { ! } & \text { PROT }\end{array}$

alkane update, adm jr., 3/2/92

CG2O1 NG2SO CG2R61 50.00

120.00 ! FNT, from CG2O1 NG2S1

CG2R61, PENALTY $=10$

CG2O1 NG2S0 CG311

CG331, PENALTY $=1.5$

42.00 119.50 ! FNT, from CG2O1 NG2SO

CG2R61 NG2SO CG311 42.00 119.50 ! fnt ga, from CG2O1 NG2S0 CG331, PENALTY $=31.5$ ! SAMO BEFORE $42.00 \quad \overline{1} 19.50$

CG324 NG3P1 CG324 45.00 115.20 ! FLAVOP PIP1,2,3 ! tweaked $115.50-->115.20$ by kevo
CG324 NG3P1 HGP2
30.00
110.80
27.00
2.07400 ! FLAVOP

PIP1, 2, 3

DIHEDRALS 


$\begin{array}{lllllll}\text { NG2S0 CG2O1 } & \text { CG321 } & \text { CG331 } & 1.5000 & 1 & 0.00 & \text { ! DMPR, } \\ \text { dimethylpropanamide, } & \text { mnoon } & & & & \\ \text { NG2S0 CG2O1 CG321 } & \text { HGA2 } & 0.0000 & 1 & 0.00 & \text { ! } & \text { PROT }\end{array}$

ACProNH2, ProNH2, ACProNHCH3 RLD 4/23/93

OG2D1 CG2O1 CG321 CG331 0.05006180 .00 ! PROT For side chains of asp, asn,glu,gln, $(n=6)$ from KK(LK)

OG2D1 CG2O1 CG321 HGA2 0.00003180 .00 ! PROT adm jr.,

$8 / 13 / 90$ geometry and vibrations

CG321 CG2O1 NG2SO CG2R61 1.6000110 .00 ! FNT ， from

CG321 CG2O1 NG2S1 CG2R61, PENALTY $=10$

CG321 CG2O1 NG2S0 CG2R61 2.5000 2 180.00 ! FNT ，from

CG321 CG2O1 NG2S1 CG2R61, PENALTY $=10$

CG321 CG2O1 NG2S0 CG311 2.6000 2 180.00 ! FNT， from

CG321 CG2O1 NG2S0 CG331, PENALTY $=1.5$

OG2D1 CG2O1 NG2S0 CG2R61 2.5000 2 180.00 ! FNT， from

OG2D1 CG2O1 NG2S1 CG2R61, PENALTY $=10$

OG2D1 CG2O1 NG2S0 CG311 2.6000 2 180.00 ! FNT ， from

OG2D1 CG2O1 NG2S0 CG331, PENALTY $=1.5$

CG2R61 CG2R61 CG2R61 CG2R61 3.1000 2 180.00 ! PROT JES

$8 / 25 / 89$

CG2R61 CG2R61 CG2R61 CG321 3.1000 2 180.00 ! PROT JES

8/25/89 toluene and ethylbenzene

CG2R61 CG2R61 CG2R61 NG2S0 3.10002 180.00 ! FNT , from

CG2R61 CG2R61 CG2R61 NG2S1, PENALTY= 1

CG2R61 CG2R61 CG2R61 HGR61 4.2000 $2 \quad 180.00$ ! PROT JES

8/25/89 benzene

CG321 CG2R61 CG2R61 HGR61 2.4000 2 180.00 ! PROT JES

8/25/89 toluene and ethylbenzene Kenno: $4.2->2.4$

NG2S0 CG2R61 CG2R61 HGR61 2.4000 2 180.00 ! FNT , from

NG2S1 CG2R61 CG2R61 HGR61, PENALTY $=1$

HGR61 CG2R61 CG2R61 HGR61 2.4000 $2 \quad 180.00$ ! PROT JES

$8 / 25 / 89$ benzene

CG2R61 CG2R61 CG321 CG324 $0.2300 \quad 2 \quad 180.00$ ! FNT ， from

CG2R61 CG2R61 CG321 CG314, PENALTY=0.6

CG2R61 CG2R61 CG321 HGA2 $0.00206 \quad 0.00$ ! PROT toluene,

adm jr., $3 / 7 / 92$

CG2R61 CG2R61 NG2S0 CG2O1 1.20002180 .00 ! FNT ， from

CG2R61 CG2R61 NG2S1 CG2O1, PENALTY= 10

!!!!!

!CG2R61 CG2R61 NG2S0 CG311 1.20002180 .00 ! f23_gaus , from CG2R61 CG2R61 NG2S1 CG2O1, PENALTY $=91.5$

CG2R61 CG2R61 NG2S0 CG311 1.50802180 .00 ! f23_gaus , from CG2R61 CG2R61 NG2S1 CG2O1, PENALTY= 91.5

$! ! ! ! !$

$\begin{array}{llllllll}\text { CG321 CG311 CG321 } & \text { CG324 } & 0.2000 & 3 & 0.00 & \text { ! G4MP, Gamma- }\end{array}$ 4-Methyl piperidine Glu Acid CDCA Amide, cacha reset to default by kevo

$\begin{array}{llllllll}\text { CG321 CG311 } & \text { CG321 } & \text { HGA2 } & 0.1950 & 1 & 0.00 & \text { ! NA, sugar }\end{array}$ NG2S0 CG311 CG321 CG324 $0.2000 \quad 3 \quad 0.00$ ! FNT ， from

NG2S1 CG311 CG321 CG324, PENALTY= 1 


\begin{tabular}{|c|c|c|c|c|c|c|c|c|}
\hline $\begin{array}{l}\text { NG2S } 0 \\
\text { NG2S } 1\end{array}$ & $\begin{array}{c}\text { CG311 } \\
\text { CG311 }\end{array}$ & $\begin{array}{r}\text { CG321 } \\
\text { G321 H }\end{array}$ & $\begin{array}{l}\text { HGA2 } \\
\text { A2, PEI }\end{array}$ & $\begin{aligned} & 0.2000 \\
= & 1\end{aligned}$ & 3 & 0.00 & ! FNT, & from \\
\hline $\begin{array}{l}\text { HGA1 } \\
\text { cacha }\end{array}$ & CG311 & CG321 & CG324 & 0.1950 & 3 & 0.00 & ! G4MP, & 01OHO3, \\
\hline HGA & CG311 & CG321 & HGA2 & 0.1950 & 3 & 0.00 & ! NA, s & pugar \\
\hline 21 & CG311 & NG2SO & $\mathrm{CG} 201$ & 1.8000 & 1 & 0.00 & ! FNT, & from \\
\hline
\end{tabular}

! ! ! ! ! ! ! ! ! ! ! ! ! ! ! ! ! ! ! ! ! ! ! ! ! ! ! ! ! ! ! ! ! ! ! ! ! ! ! ! ! ! ! ! ! ! ! ! ! ! ! ! ! ! ! ! ! ! ! !!!!!!!!!

! BEFORE: CG321 CG311 NG2SO CG2R61 $1.80001 \quad 0.00$ ! fnt_ga, from CG321 CG311 NG2S1 CG2O1, PENALTY $=40$

$\begin{array}{llllll}\text { CG321 } & \text { CG311 NG2S0 } & \text { CG2R61 } & 0.9010 & 1 & 0.00\end{array}$

$\begin{array}{llllll}\text { CG321 CG311 NG2S0 CG2R61 } & 0.5700 & 2 & 180.00\end{array}$

$\begin{array}{llllll}\text { CG321 } & \text { CG311 NG2S0 } & \text { CG2R61 } & 0.9840 & 3 & 0.00\end{array}$

$\begin{array}{llllll}\text { CG321 CG311 NG2SO CG2R61 } & 0.1050 & 6 & 180.00\end{array}$

! ! ! ! ! ! ! ! ! ! ! ! ! ! ! ! ! ! ! ! ! ! ! ! ! ! ! ! ! ! ! ! ! ! ! ! ! ! ! ! ! ! ! ! ! ! ! ! ! !

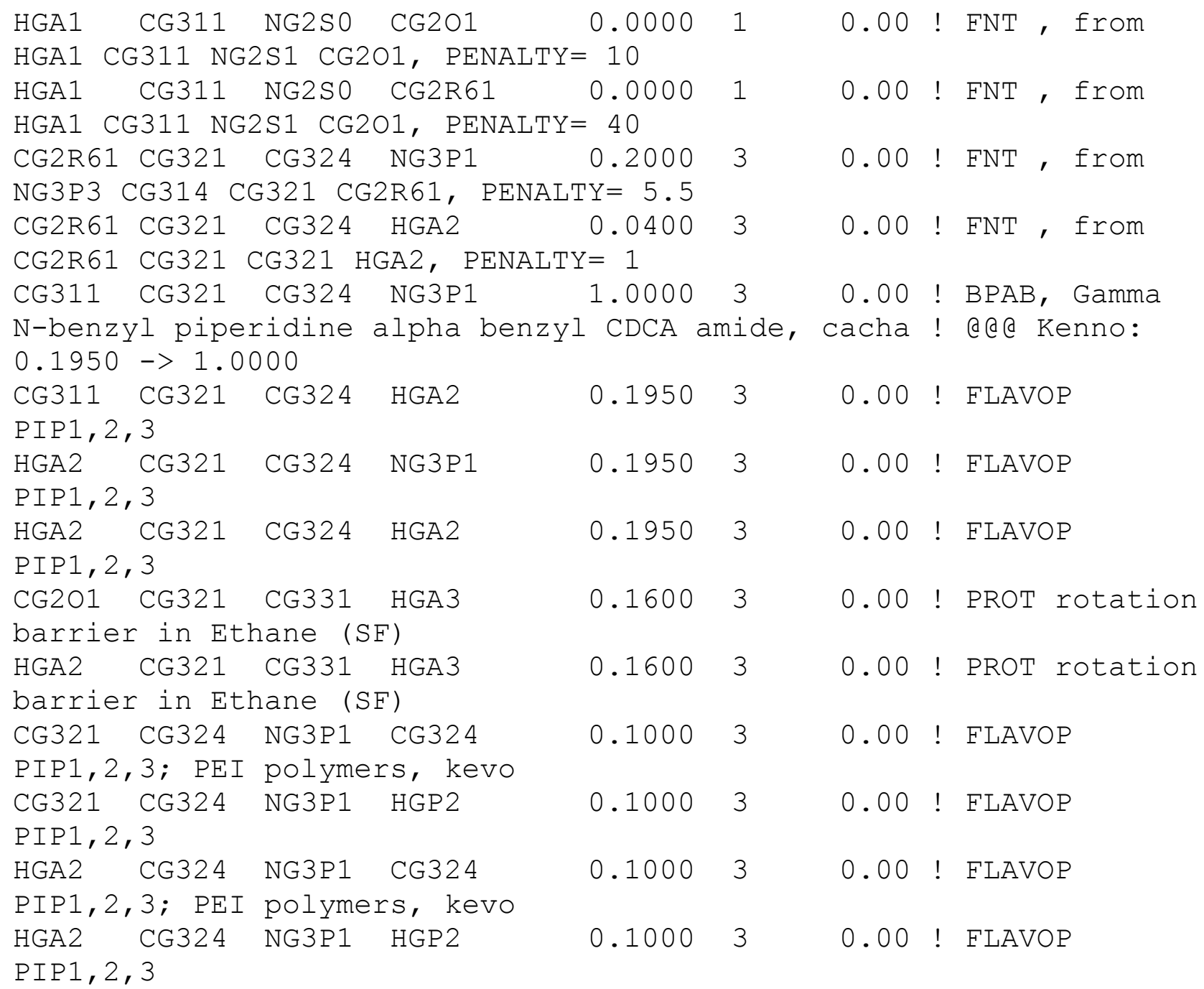

IMPROPERS 
$\begin{array}{llllllllll}\text { CG2O1 CG321 NG2S0 OG2D1 } & 120.0000 & 0 & 0.00 & \text { ! PROT NMA }\end{array}$ Vibrational Modes (LK) WILDCARD

END

RETURN 
* Topology file (.rtf) for FENTANYL (FNT); parameterization by good water interaction partial charges combined with chemical intuition; set C

* Prepared by Dr. Samo Lesnik, 2019, A. N. Bondar Group

* Potential energy function for fentanyl-based pain killers - Samo Lešnik, Milan Hodošček, Urban Bren, Christoph Stein and AnaNicoleta Bondar

*

\begin{tabular}{|c|c|c|}
\hline RESI & ENT & $1.000 !$ \\
\hline GROUP & & ! CHARGE \\
\hline ATOM & C1 CG2R61 & -0.115 \\
\hline $\mathrm{ATOM}$ & HGR 61 & 0.115 \\
\hline TOM & C2 CG2R61 & -0.108 \\
\hline ГОМ & HGR 61 & 0.115 \\
\hline TOM & C3 CG2R61 & -0.118 \\
\hline $\mathrm{OM}$ & HGR 61 & 0.115 \\
\hline ГОM & C4 CG2R61 & -0.006 \\
\hline TOM & C5 CG2R61 & -0.118 \\
\hline TOM & HGR 61 & 0.115 \\
\hline $\mathrm{TOM}$ & C6 CG2R61 & -0.108 \\
\hline TOM & H30 HGR61 & 0.115 \\
\hline $\mathrm{TOM}$ & C7 CG321 - 0 & 134 \\
\hline TOM & HGA2 & 0.090 \\
\hline АTOM & HGA 2 & 0.090 \\
\hline ATOM & C8 CG324 & 180 \\
\hline $\mathrm{OM}$ & H33 HGA2 & 0.090 \\
\hline TOM & HGA2 & 0.090 \\
\hline ATOM & N9 NG3P1 - 0. & 786 \\
\hline $\mathrm{ATOM}$ & HGP 2 & 0.344 \\
\hline TOM & CG324 & 0.314 \\
\hline TOM & $\mathrm{HGA} 2$ & 0.090 \\
\hline $\mathrm{ATOM}$ & HGA2 & 0.090 \\
\hline ATOM & CG321 - & -0.010 \\
\hline ATOM & HGA2 & 0.090 \\
\hline АTOM & HGA 2 & 0.090 \\
\hline ATOM & CG311 & 0.166 \\
\hline ATOM & HGA1 & 0.090 \\
\hline ATOM & CG321 - & -0.010 \\
\hline ATOM & HGA2 & 0.090 \\
\hline ATOM & HGA2 & 0.090 \\
\hline ATOM & CG324 & 0.314 \\
\hline ATOM & HGA2 & 0.090 \\
\hline ATOM & HGA2 & 0.090 \\
\hline ATOM & NG2SO & -0.896 \\
\hline ATOM & CG2R 61 & 0.321 \\
\hline ATOM & CG2R61 & -0.121 \\
\hline ATOM & HGR 61 & 0.115 \\
\hline ATOM & CG2R61 & -0.1 \\
\hline АTOM & HGR 61 & 0.115 \\
\hline
\end{tabular}




\begin{tabular}{|c|c|c|c|}
\hline ATOM & C19 & CG2R61 & -0.115 \\
\hline ATOM & H4 6 & HGR 61 & 0.115 \\
\hline ATOM & $\mathrm{C} 20$ & CG2R61 & -0.109 \\
\hline ATOM & H 47 & HGR 61 & 0.115 \\
\hline ATOM & $\mathrm{C} 21$ & CG2R61 & -0.121 \\
\hline ATOM & H 48 & HGR 61 & 0.115 \\
\hline ATOM & $\mathrm{C} 22$ & $\mathrm{CG} 2 \mathrm{O} 1$ & 0.200 \\
\hline ATOM & C23 & CG321 & -0.105 \\
\hline ATOM & H4 9 & HGA 2 & 0.090 \\
\hline ATOM & H5O & HGA 2 & 0.090 \\
\hline ATOM & $\mathrm{C} 24$ & CG331 & -0.270 \\
\hline ATOM & H52 & HGA 3 & 0.090 \\
\hline ATOM & H5 3 & HGA 3 & 0.090 \\
\hline ATOM & H5 4 & HGA 3 & 0.090 \\
\hline ATOM & 025 & OG2D1 & -0.250 \\
\hline BOND & $\mathrm{C} 1$ & $\mathrm{C} 2$ & \\
\hline BOND & $\mathrm{C} 2$ & C3 & \\
\hline BOND & C3 & $\mathrm{C} 4$ & \\
\hline BOND & $\mathrm{C} 4$ & $\mathrm{C} 5$ & \\
\hline BOND & $\mathrm{C} 5$ & $\mathrm{C} 6$ & \\
\hline BOND & C6 & $\mathrm{C} 1$ & \\
\hline BOND & $\mathrm{C} 7$ & C4 & \\
\hline BOND & C8 & C7 & \\
\hline BOND & N9 & $\mathrm{C} 8$ & \\
\hline BOND & $\mathrm{C} 10$ & N9 & \\
\hline BOND & $\mathrm{C} 11$ & $\mathrm{C} 10$ & \\
\hline BOND & $\mathrm{C} 12$ & $\mathrm{C} 11$ & \\
\hline BOND & C13 & $\mathrm{C} 12$ & \\
\hline BOND & $\mathrm{C} 14$ & N9 & \\
\hline BOND & N15 & $\mathrm{C} 12$ & \\
\hline BOND & $\mathrm{C} 16$ & N15 & \\
\hline BOND & $\mathrm{C} 17$ & C16 & \\
\hline BOND & C18 & C17 & \\
\hline BOND & C19 & C18 & \\
\hline BOND & $\mathrm{C} 20$ & C19 & \\
\hline BOND & $\mathrm{C} 21$ & C16 & \\
\hline BOND & C22 & N15 & \\
\hline BOND & C23 & $\mathrm{C} 22$ & \\
\hline BOND & C24 & $\mathrm{C} 23$ & \\
\hline BOND & 025 & $\mathrm{C} 22$ & \\
\hline BOND & H2 6 & $\mathrm{C} 1$ & \\
\hline BOND & H2 7 & $\mathrm{C} 2$ & \\
\hline BOND & H2 8 & C3 & \\
\hline BOND & H2 9 & C5 & \\
\hline BOND & H3O & $\mathrm{C} 6$ & \\
\hline BOND & H31 & C7 & \\
\hline BOND & H32 & C7 & \\
\hline BOND & H33 & $\mathrm{C} 8$ & \\
\hline BOND & H3 4 & $\mathrm{C} 8$ & \\
\hline BOND & H3 5 & $\mathrm{C} 10$ & \\
\hline BOND & H3 6 & C10 & \\
\hline BOND & H37 & C11 & \\
\hline
\end{tabular}




$\begin{array}{llll}\text { BOND } & \text { H38 } & \text { C11 } \\ \text { BOND } & \text { H39 } & \text { C12 } \\ \text { BOND } & \text { H40 } & \text { C13 } \\ \text { BOND } & \text { H41 } & \text { C13 } \\ \text { BOND } & \text { H42 } & \text { C14 } \\ \text { BOND } & \text { H43 } & \text { C14 } \\ \text { BOND } & \text { H44 } & \text { C17 } \\ \text { BOND } & \text { H45 } & \text { C18 } \\ \text { BOND } & \text { H46 } & \text { C19 } & \\ \text { BOND } & \text { H47 } & \text { C20 } & \\ \text { BOND } & \text { H48 } & \text { C21 } & \\ \text { BOND } & \text { H49 } & \text { C23 } & \\ \text { BOND } & \text { H50 } & \text { C23 } & \\ \text { BOND } & \text { H51 } & \text { N9 } & \\ \text { BOND } & \text { C13 } & \text { C14 } & \\ \text { BOND } & \text { C20 } & \text { C21 } & \\ \text { BOND } & \text { H52 } & \text { C24 } & \\ \text { BOND } & \text { H53 } & \text { C24 } & \\ \text { BOND } & \text { H54 } & \text { C24 } & \\ \text { IMPR } & \text { C22 } & \text { C23 } & \text { N15 }\end{array}$

* Parameter file (.prm) for FENTANYL (ENT); parameterization by good water interaction partial charges combined with chemical intuition; Set C

* Prepared by Dr. Samo Lesnik, 2019, A. N. Bondar Group

* Potential energy function for fentanyl-based pain killers - Samo Lešnik, Milan Hodošček, Urban Bren, Christoph Stein and AnaNicoleta Bondar

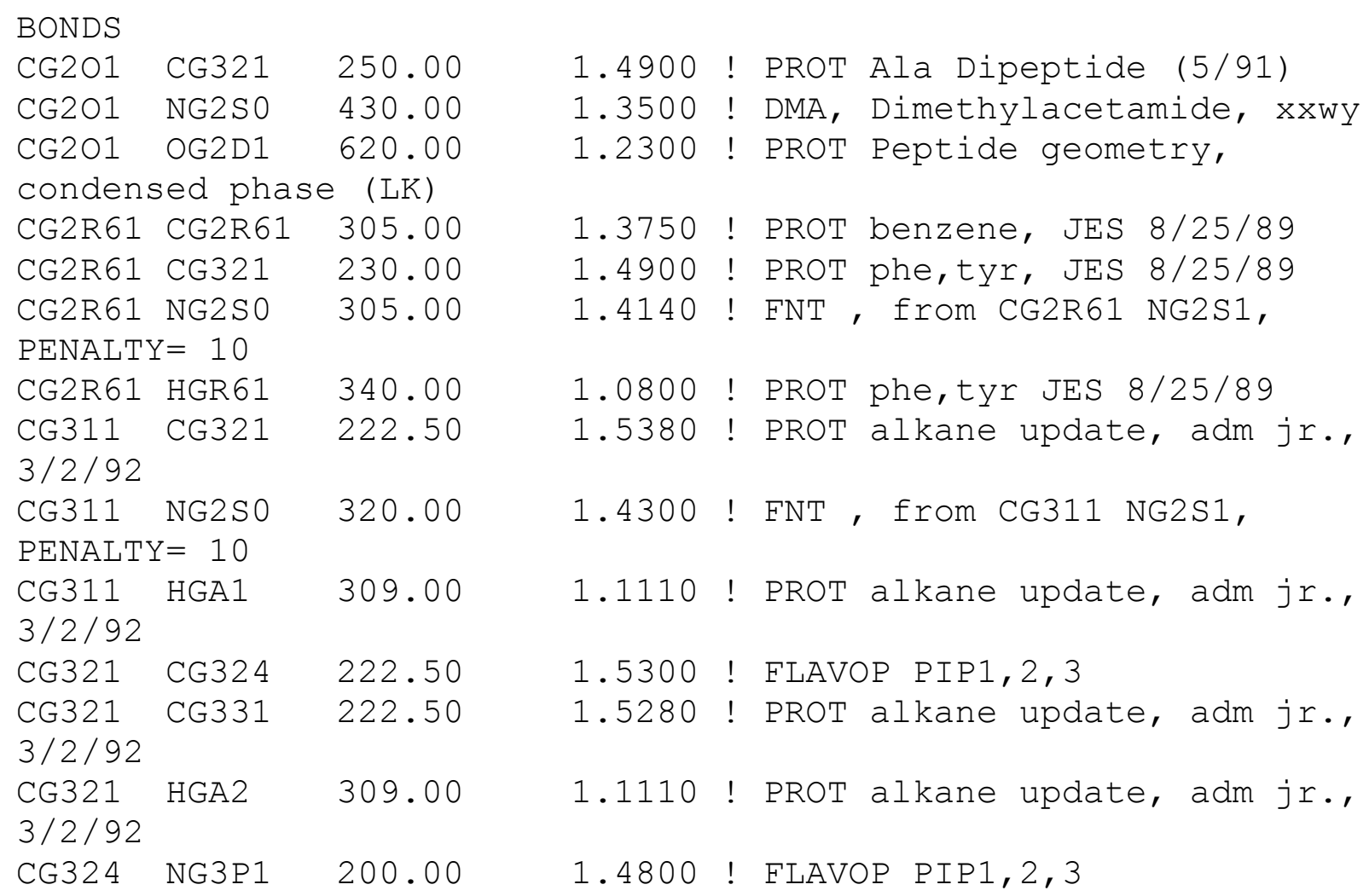




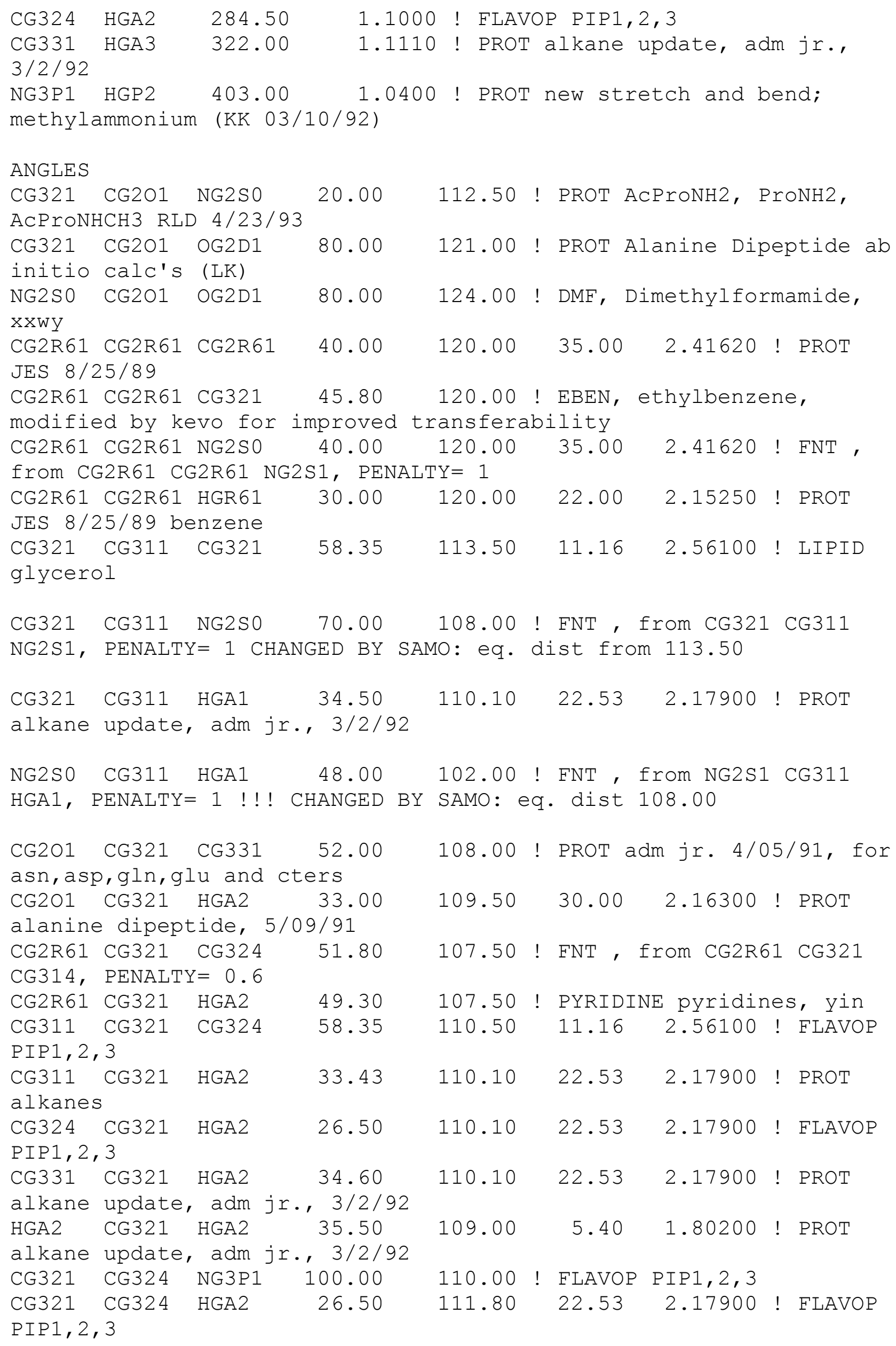




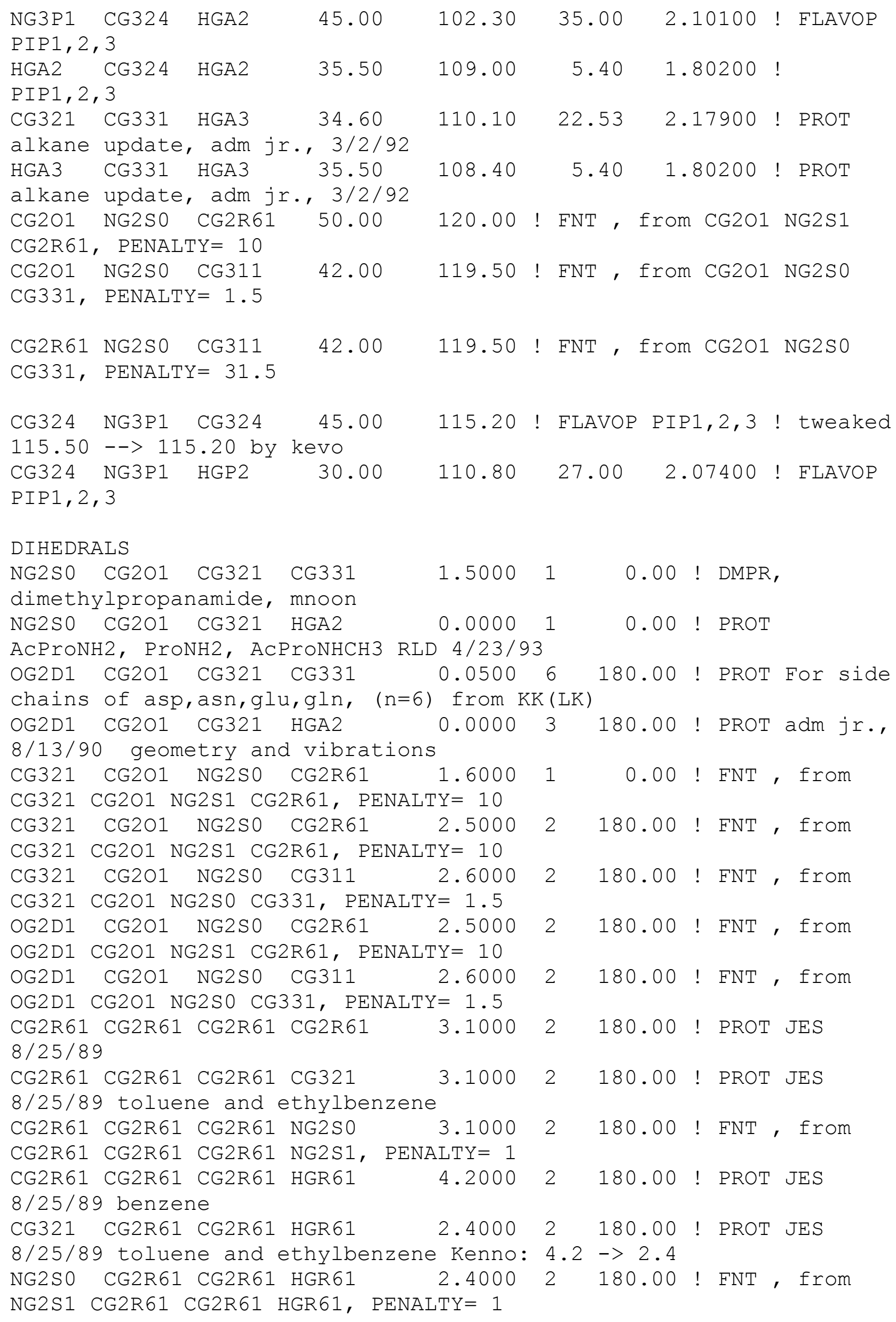


HGR61 CG2R61 CG2R61 HGR61 2.40002180 .00 ! PROT JES $8 / 25 / 89$ benzene

CG2R61 CG2R61 CG321 CG324 0.65002 180.00 ! FNT ， from CG2R61 CG2R61 CG321 CG314, PENALTY=0.6 ! SAMO: before 0.23002 180.00

CG2R61 CG2R61 CG321 HGA2 $0.00206 \quad 0.00$ ! PROT toluene, adm jr., $3 / 7 / 92$

CG2R61 CG2R61 NG2S0 CG2O1 1.20002180 .00 ! FNT ， from CG2R61 CG2R61 NG2S1 CG2O1, PENALTY= 10

!!!!!!!!!!!!!!!!!!!!!!!!!!!!!

!CG2R61 CG2R61 NG2S0 CG311 1.20002180 .00 ! fnt_gaus , from CG2R61 CG2R61 NG2S1 CG2O1, PENALTY= 91.5

$\begin{array}{llllll}\text { CG2R61 CG2R61 NG2S0 CG311 } & 1.7260 & 2 & 180.00\end{array}$

$\begin{array}{llllll}\text { CG2R61 CG2R61 NG2S0 CG311 } & 1.9090 & 1 & 180.00\end{array}$

! ! ! ! ! ! ! ! ! ! ! ! ! ! ! ! ! ! ! ! !

CG321 CG311 CG321 CG324 $0.2000 \quad 3 \quad 0.00$ ! G4MP, Gamma4-Methyl piperidine Glu Acid CDCA Amide, cacha reset to default by kevo

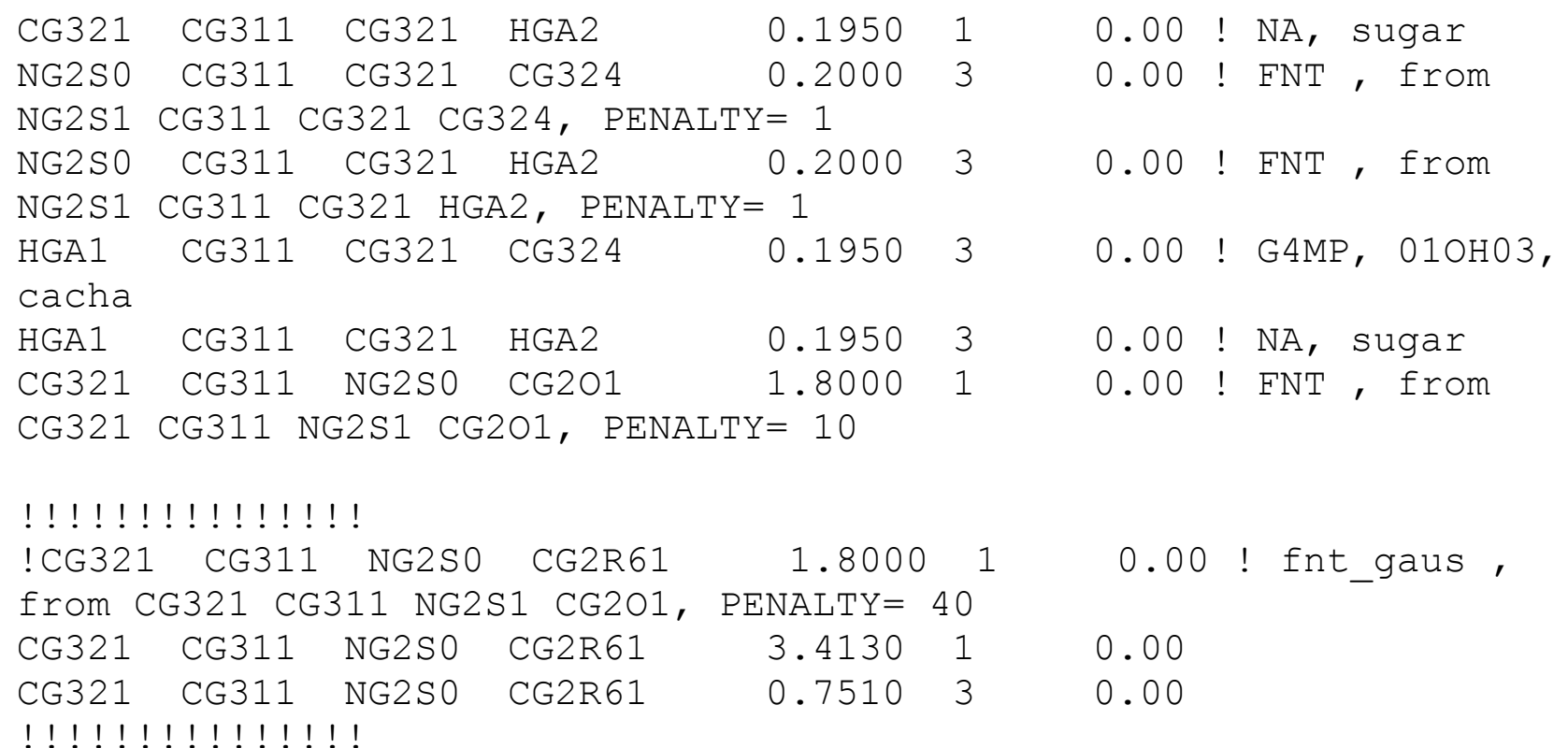

HGA1 CG311 NG2SO CG2O1 0.0000110 .00 ! FNT， from HGA1 CG311 NG2S1 CG2O1, PENALTY $=10$

HGA1 CG311 NG2S0 CG2R61 0.0000110 .00 ! FNT， from HGA1 CG311 NG2S1 CG2O1, PENALTY $=40$

CG2R61 CG321 CG324 NG3P1 0.200030 .00 ! FNT , from

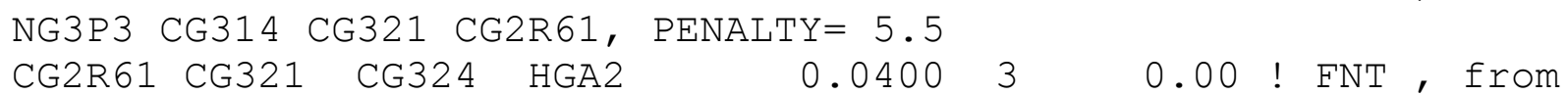
CG2R61 CG321 CG321 HGA2, PENALTY= 1 


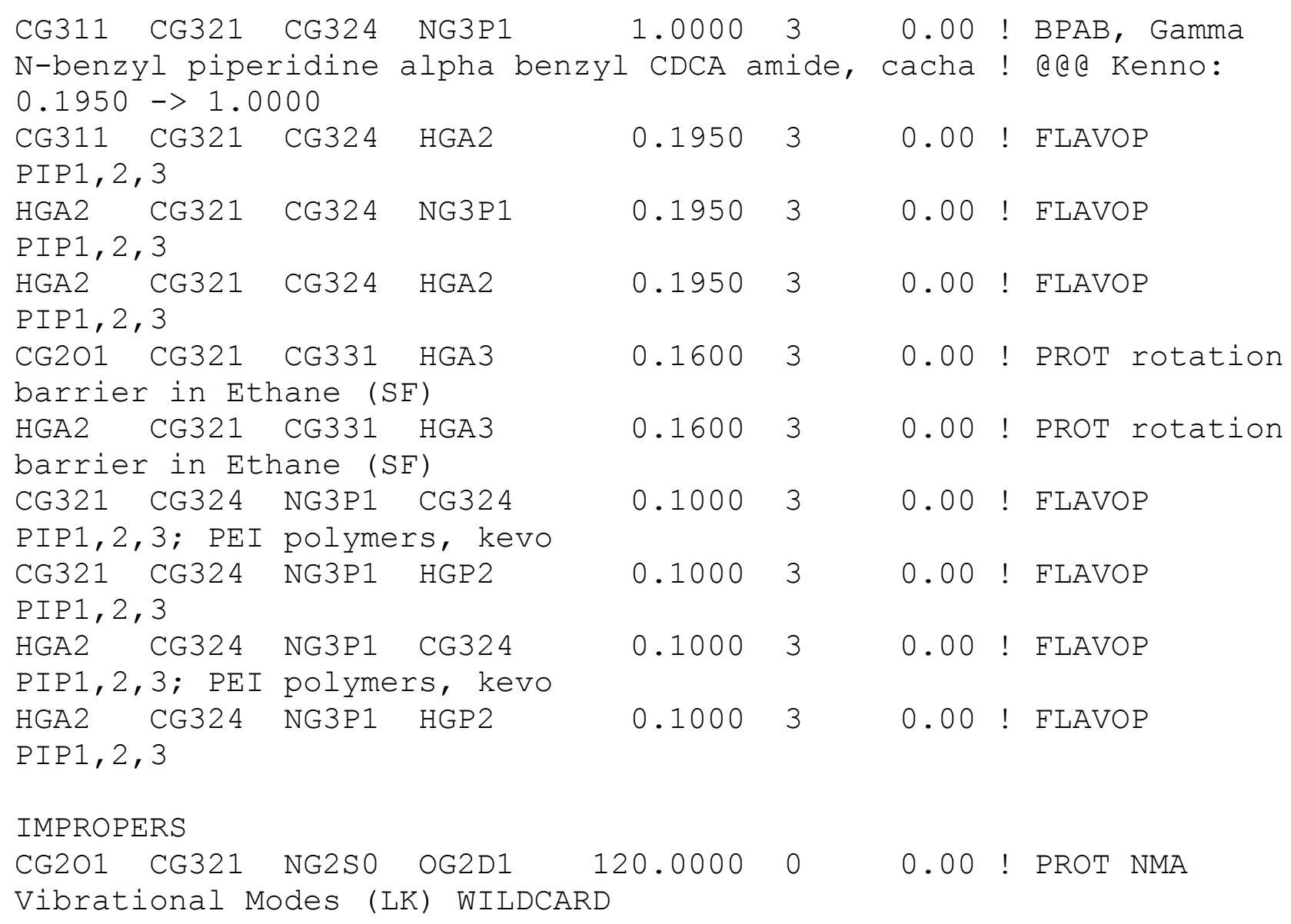




\section{NFEPP parameters}

* Topology file for NFEPP (3FP);

* Prepared by Dr. Samo Lesnik, 2019, A. N. Bondar Group

* Potential energy function for fentanyl-based pain killers - Samo Lešnik, Milan Hodošček, Urban Bren, Christoph Stein and AnaNicoleta Bondar

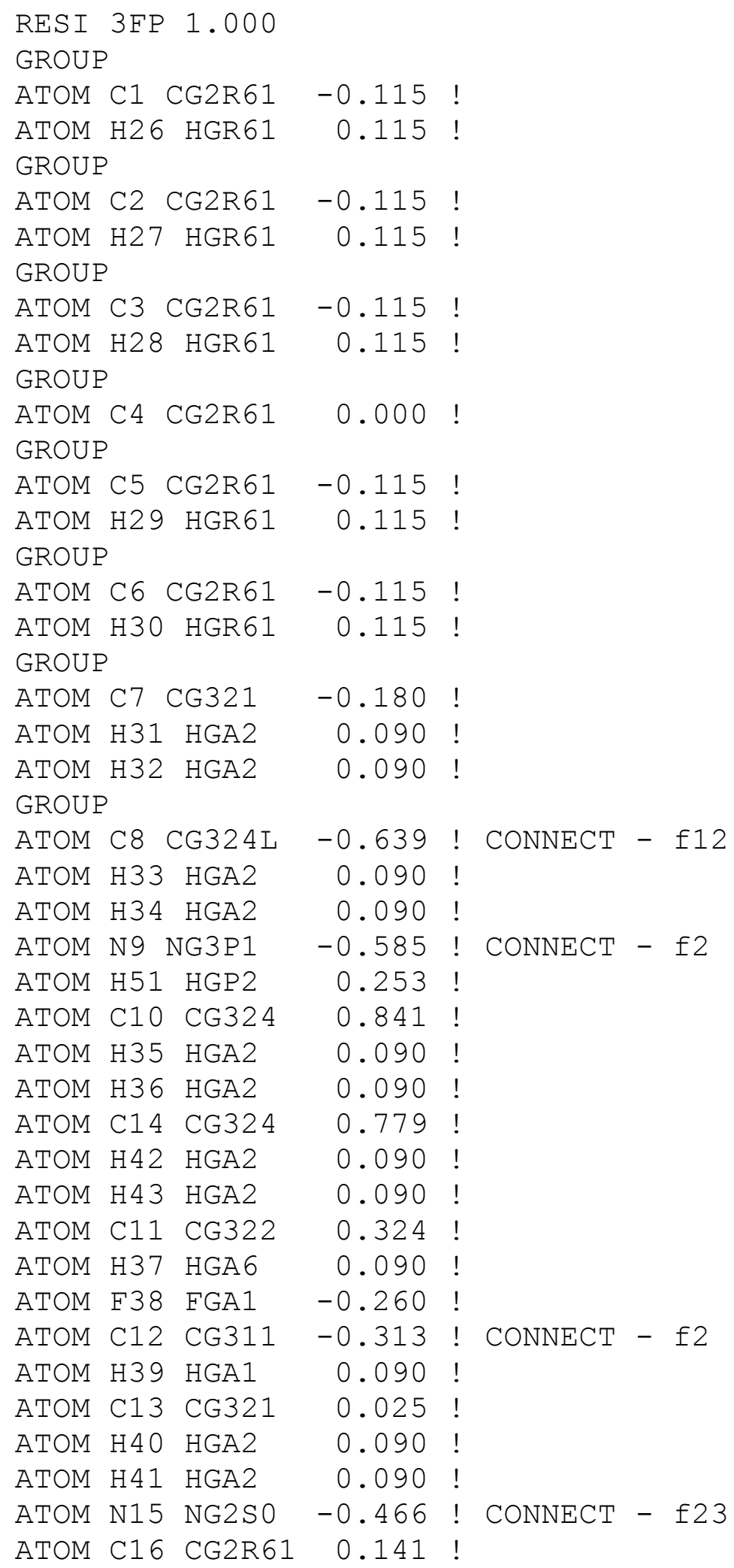




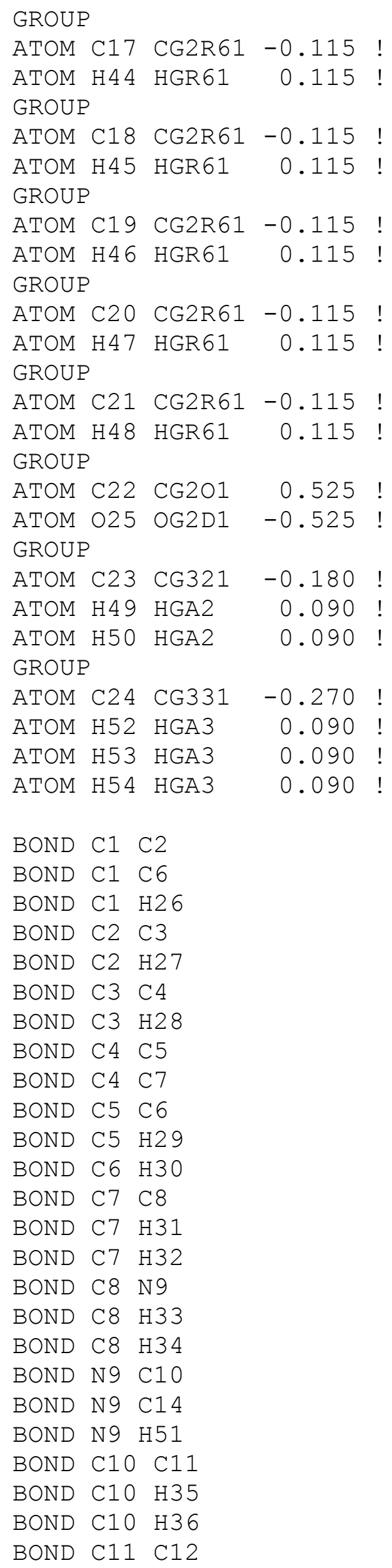




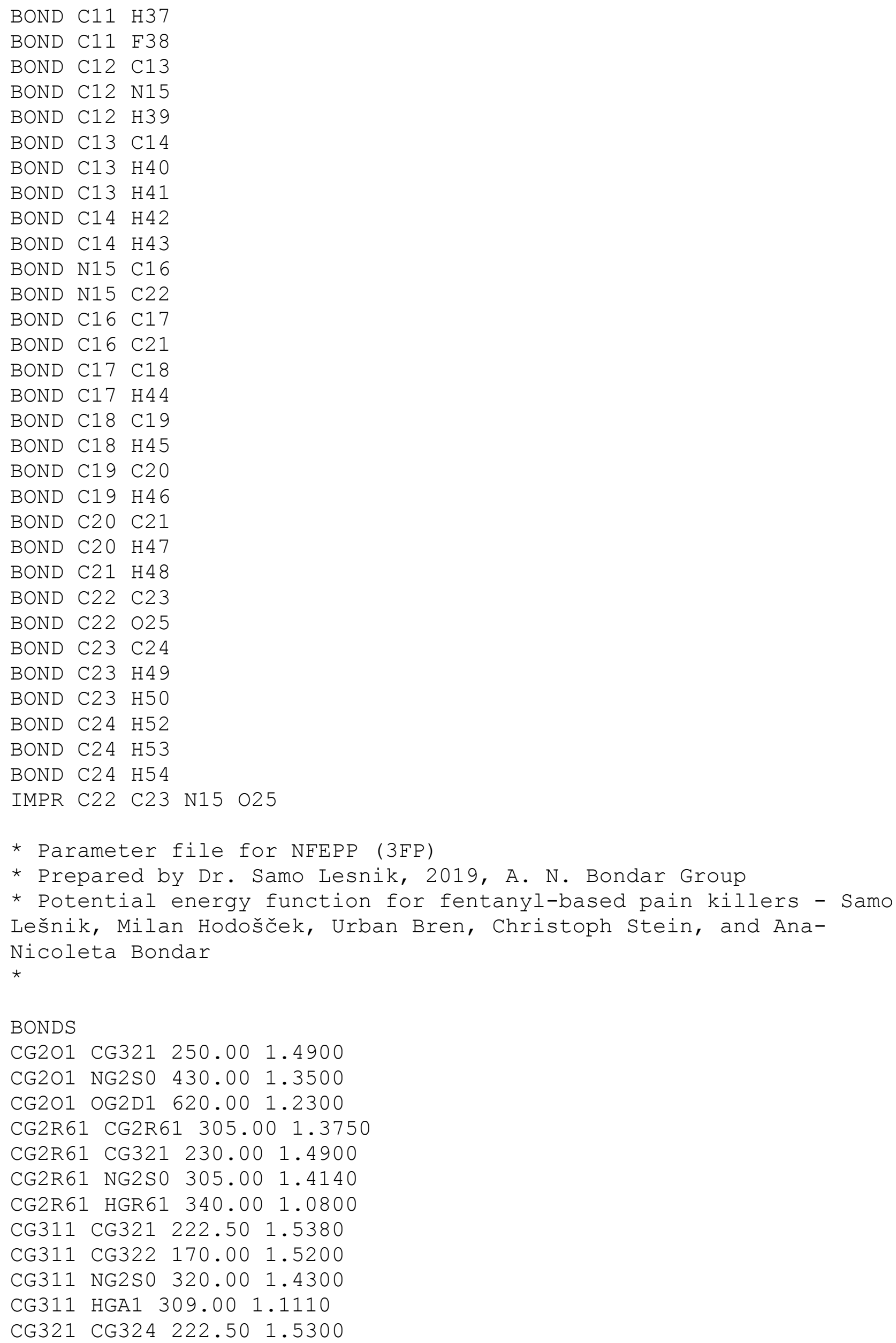


CG321 CG331 222.50 1.5280

CG321 HGA2 $309.00 \quad 1.1110$

CG322 CG324 170.001 .5200

CG322 FGA1 420.001 .3740

CG322 HGA 6342.001 .0828

CG324 NG3P1 200.00 1.4800

CG324 HGA2 $284.50 \quad 1.1000$

CG331 HGA3 322.00 1.1110

NG3P1 HGP2 403.00 1.0400

! ! ! ! ! ! ! ! ! ! ! ! NEW TYPE C8: CG324L ! ! ! ! ! !

CG321 CG324L 222.50 1.5300

CG322 CG324L 170.001 .5200

CG324L NG3P1 200.00 1.4800

CG324L HGA2 284.501 .1000

! ! ! ! ! ! ! ! ! ! ! ! End New Type ! ! ! ! ! ! ! ! ! ! ! !

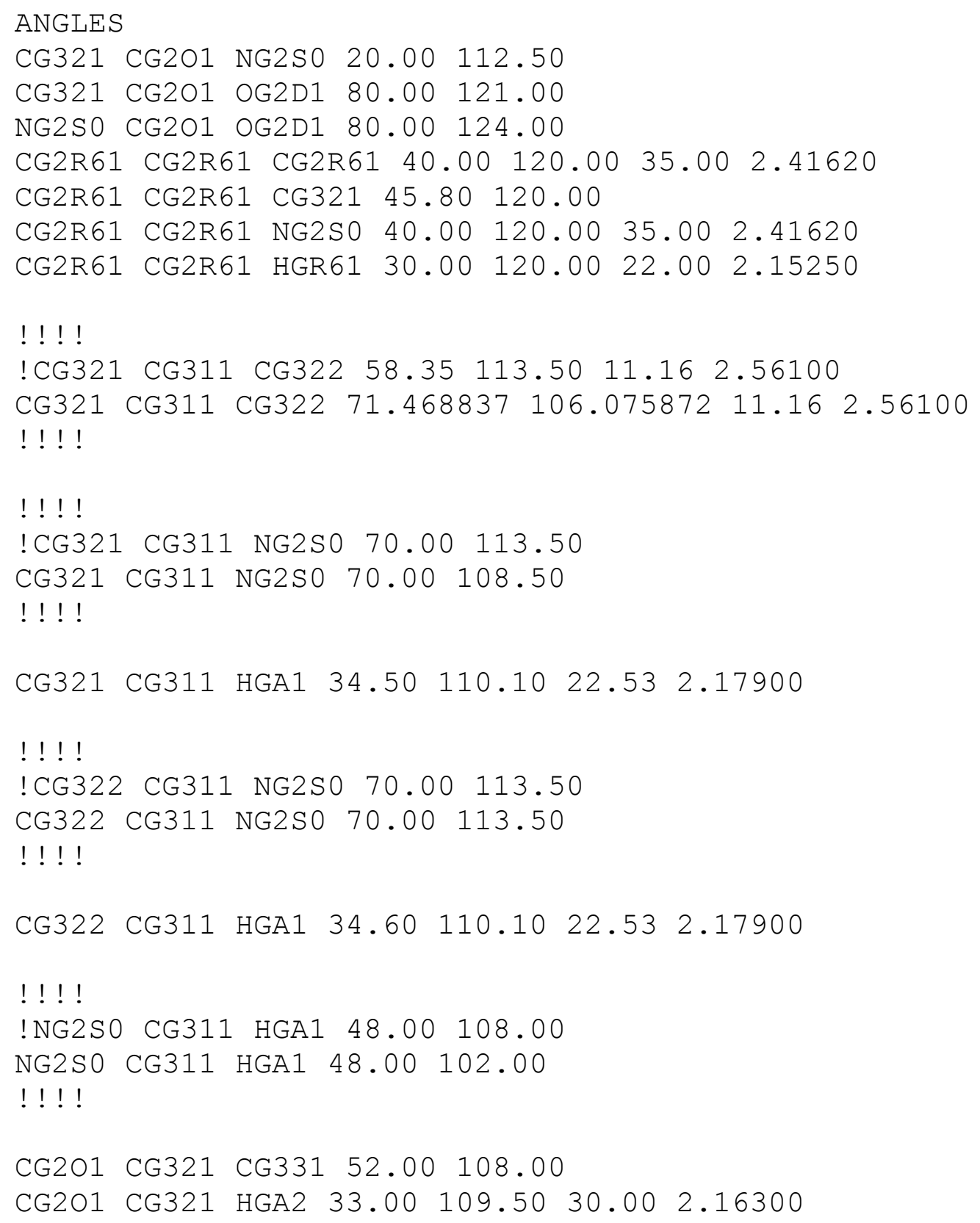


CG2R61 CG321 CG324 $51.80 \quad 107.50$

CG2R61 CG321 HGA2 49.30107 .50

CG311 CG321 CG324 $58.35 \quad 110.50 \quad 11.16 \quad 2.56100$

CG311 CG321 HGA2 $33.43 \quad 110.10 \quad 22.53 \quad 2.17900$

CG324 CG321 HGA2 $26.50 \quad 110.10 \quad 22.53 \quad 2.17900$

CG331 CG321 HGA2 $34.60110 .10 \quad 22.53 \quad 2.17900$

HGA2 CG321 HGA2 $35.50109 .00 \quad 5.401 .80200$

\section{!! ! !}

$\begin{array}{llllllll}\text { !CG311 CG322 CG324 } 44.00 & 112.00 & 30.00 & 2.36900\end{array}$

$\begin{array}{lllllllll}\text { CG311 CG322 CG324 } 22.350537 & 118.332339 & 30.00 & 2.36900\end{array}$

!!!!!

CG311 CG322 FGA1 $44.00 \quad 112.00 \quad 30.00 \quad 2.36900$

CG311 CG322 HGA6 $31.00 \quad 112.00 \quad 3.00 \quad 2.16800$

!!!!

!CG324 CG322 FGA1 44.00 $112.00 \quad 30.00 \quad 2.36900$

CG324 CG322 FGA1 27.435839 $101.695970 \quad 30.00 \quad 2.36900$

!!!!

\section{!!!!!}

!CG324 CG322 HGA6 $31.00 \quad 112.00 \quad 3.00 \quad 2.16800$

CG324 CG322 HGA6 46.776011 $98.246447 \quad 3.00 \quad 2.16800$

!!!!

FGA1 CG322 HGA6 57.50 $108.895 .00 \quad 1.99700$

CG321 CG324 NG3P1 100.00110 .00

CG321 CG324 HGA2 $26.50 \quad 111.80 \quad 22.53 \quad 2.17900$

\section{!! ! ! !}

!CG322 CG324 NG3P1 100.00110 .00

CG322 CG324 NG3P1 79.037519110 .680616

!!!!!

$\begin{array}{llllllll}\text { CG322 CG324 HGA2 } & 34.60 & 110.10 & 22.53 & 2.17900\end{array}$

NG3P1 CG324 HGA2 $45.00 \quad 102.30 \quad 35.00 \quad 2.10100$

HGA2 CG324 HGA2 $35.50 \quad 109.00 \quad 5.40 \quad 1.80200$

$\begin{array}{llllllllll}\text { CG321 CG331 HGA3 } & 34.60 & 110.10 & 22.53 & 2.17900\end{array}$

HGA3 CG331 HGA3 $35.50 \quad 108.40 \quad 5.40 \quad 1.80200$

CG2O1 NG2SO CG2R61 $50.00 \quad 120.00$

CG2O1 NG2SO CG311 $42.00 \quad 119.50$

$! ! ! !$

!CG2R61 NG2S0 CG311 $42.00 \quad 119.50$

CG2R61 NG2S0 CG311 $42.00 \quad 119.50$

!!! !

CG324 NG3P1 CG324 $45.00 \quad 115.20$

CG324 NG3P1 HGP2 $30.00 \quad 110.80 \quad 27.00 \quad 2.07400$ 
!! !!!!!!!!!!!!! ! NEW TYPE C8 :

CG324L ! ! ! ! ! ! ! ! ! ! ! ! ! ! ! ! ! ! ! ! ! ! ! ! ! ! ! ! ! ! ! ! ! ! ! ! ! ! ! ! ! ! ! ! ! ! ! ! ! !

! ! ! ! ! ! ! ! ! ! ! ! ! ! ! ! ! ! ! ! ! ! ! ! !

CG2R61 CG321 CG324L 51.800 102.405 ! Before:

CG2R61 CG321 CG324 $51.800 \quad 107.500$

$\begin{array}{lllllll}\text { CG324L } & \text { CG321 HGA2 } & 26.500 & 97.925 & 22.53 & 2.1790 & \text { ! }\end{array}$

Before: CG324 CG321 HGA2 26.500 $110.100 \quad 22.532 .1790$

CG321 CG324L NG3P1 $105.000 \quad 116.076$

Before: CG321 CG324 NG3P1 100.000110 .000

$\begin{array}{lllllll}\text { CG321 CG324L HGA2 } & 26.500 & 119.640 & 22.53 & 2.1790 \quad \text { ! }\end{array}$

Before: CG321 CG324 HGA2 26.500 $111.800 \quad 22.532 .1790$

NG3P1 CG324L HGA2 45.000 $107.233 \quad 35.00 \quad 2.1010$ !

Before: NG3P1 CG324 HGA2 45.000 $102.300 \quad 35.002 .1010$

HGA2 CG324L HGA2 $35.500 \quad 114.650 \quad 5.40 \quad 1.8020$ !

Before: HGA2 CG324 HGA2 35.500 109.0005 .401 .8020

CG324L NG3P1 CG324 $45.000 \quad 109.565$

Before: CG324 NG3P1 CG324 45.000 115.200

$\begin{array}{lllllll}\text { CG324L NG3P1 HGP2 } 30.000 & 111.269 & 27.00 & 2.0740 \quad \text { ! }\end{array}$

Before: CG324 NG3P1 HGP2 30.000110 .80027 .002 .0740

! ! ! ! ! ! ! ! ! ! !! End New

Type ! ! ! ! ! ! ! ! ! ! ! ! ! ! ! ! ! ! ! ! ! ! ! ! ! ! ! ! ! ! ! ! ! ! ! ! ! ! ! ! ! ! ! ! ! ! ! ! ! ! ! ! ! ! ! ! ! ! ! ! ! ! ! ! ! ! ! ! ! ! ! ! ! ! ! ! ! ! ! ! !

\author{
DIHEDRALS \\ NG2SO CG2O1 CG321 CG331 $1.5000 \quad 1 \quad 0.00$ \\ NG2SO CG2O1 CG321 HGA2 0.000010 .00 \\ OG2D1 CG2O1 CG321 CG331 $0.0500 \quad 6 \quad 180.00$ \\ OG2D1 CG2O1 CG321 HGA2 $0.0000 \quad 3 \quad 180.00$ \\ CG321 CG2O1 NG2S0 CG2R61 1.600010 .00 \\ CG321 CG2O1 NG2S0 CG2R61 2.50002180 .00 \\ CG321 CG2O1 NG2S0 CG311 2.6000 2 180.00 \\ OG2D1 CG2O1 NG2S0 CG2R61 2.5000 2180.00 \\ OG2D1 CG2O1 NG2S0 CG311 2.6000 2 180.00 \\ CG2R61 CG2R61 CG2R61 CG2R61 3.1000 2180.00 \\ CG2R61 CG2R61 CG2R61 CG321 3.10002180 .00 \\ CG2R61 CG2R61 CG2R61 NG2S0 3.10002180 .00 \\ CG2R61 CG2R61 CG2R61 HGR61 4.2000 2180.00 \\ CG321 CG2R61 CG2R61 HGR61 2.4000 2180.00 \\ NG2S0 CG2R61 CG2R61 HGR61 2.4000 2180.00 \\ HGR61 CG2R61 CG2R61 HGR61 2.4000 2180.00 \\ $\begin{array}{llllllllllll}\text { CG2R61 CG2R61 CG321 CG324 } & 0.2300 & 2 & 180.00\end{array}$ \\ CG2R61 CG2R61 CG321 HGA2 $0.0020 \quad 6 \quad 0.00$ \\ CG2R61 CG2R61 NG2S0 CG2O1 1.20002180 .00 \\ $! ! ! !$ \\ !CG2R61 CG2R61 NG2S0 CG311 1.20002180 .00 \\ $\begin{array}{lllllllllll}\text { CG2R61 CG2R61 NG2S0 CG311 } & 3.987 & 1 & 0.00\end{array}$ \\ CG2R61 CG2R61 NG2S0 CG311 2.1162180 .00 \\ CG2R61 CG2R61 NG2S0 CG311 $0.500 \quad 3 \quad 180.00$ \\ CG2R61 CG2R61 NG2S0 CG311 0.6494180 .00 \\ !!!!
}


!!!!

$\begin{array}{lllllllllllll}\text { !CG322 CG311 CG321 CG324 } & 0.2000 & 3 & 0.00\end{array}$

$\begin{array}{lllllllllllll}\text { CG322 CG311 CG321 CG324 } & 0.2180 & 3 & 0.00\end{array}$

!!!!

!!!!

CG322 CG311 CG321 HGA2 $0.1950 \quad 3 \quad 0.00$

!!!!

$\begin{array}{lllllllllll}\text { NG2SO } & \text { CG311 CG321 } & \text { CG324 } & 0.2000 & 3 & 0.00\end{array}$ NG2S0 CG311 CG321 HGA2 $0.2000 \quad 3 \quad 0.00$

$\begin{array}{llllllllllllll}\text { HGA1 CG311 } & \text { CG321 } & \text { CG324 } & 0.1950 & 3 & 0.00\end{array}$

HGA1 CG311 CG321 HGA2 $0.1950 \quad 3 \quad 0.00$

!!!!

$\begin{array}{llllllllllllll}\text { !CG321 CG311 CG322 CG324 } & 0.2000 & 3 & 0.00\end{array}$

$\begin{array}{llllllllll}\text { CG321 CG311 } & \text { CG322 } & \text { CG324 } & 2.2740 & 3 & 180.00\end{array}$

!!!!

!!!!

$\begin{array}{lllllllllll}\text { !CG321 CG311 CG322 FGA1 } 0.2500 & 3 & 0.00\end{array}$

CG321 CG311 CG322 FGA1 $0.6440 \quad 3 \quad 0.00$

!!!!

!!!!

CG321 CG311 CG322 HGA6 $0.1950 \quad 3 \quad 0.00$

!!!!

$! ! ! !$

$\begin{array}{llllllllll} & \text { NG2SO } & \text { CG311 } & \text { CG322 } & \text { CG324 } & 0.2000 & 3 & 0.00\end{array}$

NG2SO CG311 CG322 CG324 $3.464 \quad 1 \quad 0.00$

NG2SO CG311 CG322 CG324 1.47922180 .00

NG2SO CG311 CG322 CG324 $2.742 \quad 3 \quad 180.00$

NG2S0 CG311 CG322 CG324 1.0494180 .00

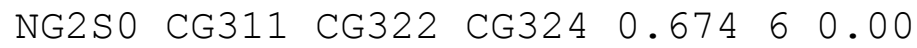

! ! !

$! ! ! !$

!NG2S0 CG311 CG322 FGA1 $0.2000 \quad 3 \quad 0.00$

$\begin{array}{llllllllll}\text { NG2SO } & \text { CG311 CG322 } & \text { FGA1 } & 0.587 & 1 & 0.00\end{array}$

NG2S0 CG311 CG322 FGA1 $4.616 \quad 3 \quad 180.00$

NG2S0 CG311 CG322 FGA1 $1.965 \quad 4 \quad 180.00$

NG2S0 CG311 CG322 FGA1 $1.273 \quad 6 \quad 180.00$

!! ! !

$! ! ! !$

NG2SO CG311 CG322 HGA6 $6.031 \quad 3 \quad 0.00$

!!!!

!!!!

HGA1 CG311 CG322 CG324 $2.175 \quad 3 \quad 180.00$

!!!! 
!!!!

HGA1 CG311 CG322 FGA1 1.2693180 .00

!!!!

!!!!

HGA1 CG311 CG322 HGA6 5.806 1180.00

!!!!

CG321 CG311 NG2SO CG2O1 $1.8000 \quad 1 \quad 0.00$

\section{!!!!}

$\begin{array}{lllllllll}\text { !CG321 CG311 NG2S0 CG2R61 } & 1.8000 & 1 & 0.00\end{array}$

CG321 CG311 NG2S0 CG2R61 3.4171180 .00

CG321 CG311 NG2S0 CG2R61 0.49930 .00

CG321 CG311 NG2S0 CG2R61 0.73140 .00

!!!!

!!!!

!CG322 CG311 NG2SO CG2O1 1.800010 .00

CG322 CG311 NG2S0 CG2O1 4.365110 .00

CG322 CG311 NG2S0 CG2O1 0.55722180 .00

CG322 CG311 NG2S0 CG2O1 $0.303 \quad 3 \quad 0.00$

CG322 CG311 NG2S0 CG2O1 $0.447 \quad 4 \quad 180.00$

!!!!

!!!!

!CG322 CG311 NG2S0 CG2R61 1.800010 .00

CG322 CG311 NG2S0 CG2R61 0.9631180 .00

CG322 CG311 NG2S0 CG2R61 $1.100 \quad 2 \quad 0.00$

CG322 CG311 NG2S0 CG2R61 $0.274 \quad 3 \quad 0.00$

CG322 CG311 NG2S0 CG2R61 $0.343 \quad 4 \quad 0.00$

!!!!

HGA1 CG311 NG2S0 CG2O1 0.000010 .00

!!!!

$\begin{array}{llllllllll}\text { !HGA1 CG311 NG2S0 CG2R61 } & 0.0000 & 1 & 0.00\end{array}$ HGA1 CG311 NG2S0 CG2R61 $3.978 \quad 1 \quad 180.00$ !!!!

$\begin{array}{lllllllllllllll}\text { CG2R61 CG321 CG324 NG3P1 } & 0.2000 & 3 & 0.00\end{array}$ CG2R61 CG321 CG324 HGA2 $0.0400 \quad 3 \quad 0.00$ CG311 CG321 CG324 NG3P1 $1.0000 \quad 3 \quad 0.00$ CG311 CG321 CG324 HGA2 $0.1950 \quad 3 \quad 0.00$ $\begin{array}{llllllllllll}\text { HGA2 } & \text { CG321 } & \text { CG324 NG3P1 } & 0.1950 & 3 & 0.00\end{array}$ $\begin{array}{lllllllll}\text { HGA2 } & \text { CG321 CG324 HGA2 } & 0.1950 & 3 & 0.00\end{array}$ CG2O1 CG321 CG331 HGA3 $0.1600 \quad 3 \quad 0.00$ $\begin{array}{llllllllll}\text { HGA } 2 & \text { CG321 CG331 HGA3 } & 0.1600 & 3 & 0.00\end{array}$

!!!!

!CG311 CG322 CG324 NG3P1 1.000030 .00 CG311 CG322 CG324 NG3P1 $1.1410 \quad 3 \quad 0.00$ !!!! 


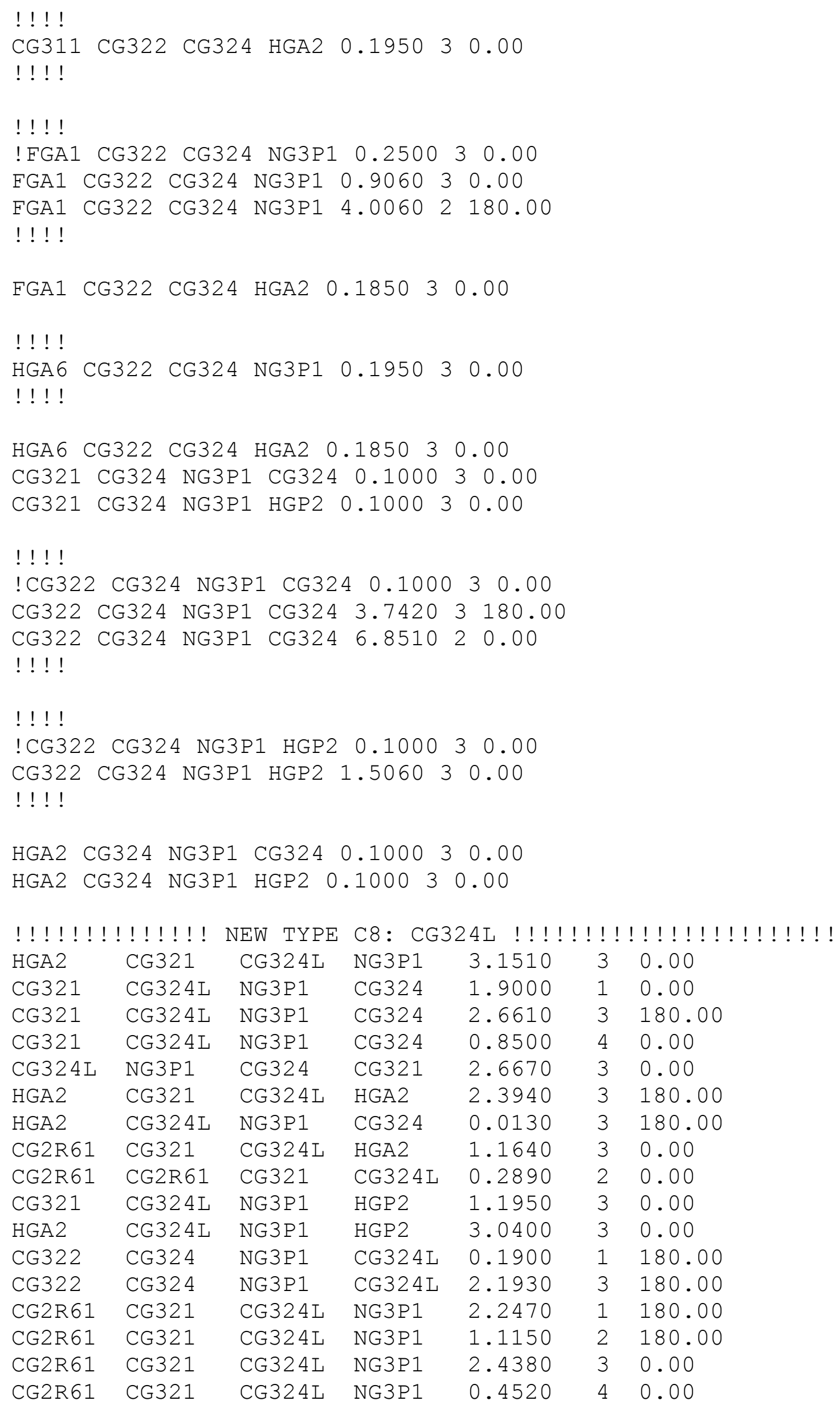


CG324L NG3P1 CG324 HGA2 $0.2480 \quad 3 \quad 180.00$

! ! ! ! ! ! ! ! ! ! ! ! ! End New Type ! ! ! ! ! ! ! ! ! ! ! ! ! ! ! ! ! ! ! ! ! ! ! !

IMPROPERS

CG2O1 CG321 NG2S0 OG2D1 120.0000 0 0.00 ! PROT NMA Vibrational Modes (LK) WILDCARD

END

RETURN 


\section{Supplementary references}

1. Peeters, O. M.; Blaton, N. M.; de Ranter, C. J.; van Herk, A. M.; Goubitz, K. Crystal and Molecular Structure of N-[1-(2-phenylethyl)-4-piperidinylium]-N-phenylpropanamide (Fentanyl) Citrate-Toluene Solvate. J. Cryst. Mol. Struct. 1979, 9, 153-161. 